\title{
Mapping Grains, Boundaries, and Defects in 2D Covalent Organic Framework Thin Films
}

\author{
Ioannina Castano ${ }^{a \neq}$, Austin M. Evans ${ }^{a \neq}$, Roberto dos Reis ${ }^{b}$, Vinayak P. Dravid ${ }^{b, c, d^{*}}$, \\ Nathan C. Gianneschi ${ }^{a, b, d, e, f, g, h^{*}}$, William R. Dichtel ${ }^{*}$ \\ ${ }^{a}$ Department of Chemistry, Northwestern University, Evanston, IL 60208, USA \\ ${ }^{\mathrm{b}}$ Department of Materials Science and Engineering, Northwestern University, Evanston, IL 60208, USA \\ ${ }^{\mathrm{c}}$ Northwestern University Atomic and Nanoscale Characterization Center, Northwestern University, Evanston, IL \\ 60208, USA \\ ${ }^{\mathrm{d} I n t e r n a t i o n a l ~ I n s t i t u t e ~ f o r ~ N a n o t e c h n o l o g y, ~ N o r t h w e s t e r n ~ U n i v e r s i t y, ~ E v a n s t o n, ~ I L ~ 60208, ~ U S A ~}$ \\ ${ }^{\mathrm{e}}$ Department of Biomedical Engineering, Northwestern University, Evanston, IL 60208, USA \\ ${ }_{\mathrm{f}}$ Department of Pharmacology, Northwestern University, Chicago, IL 60611, USA \\ ${ }^{\mathrm{g}}$ Simpson Querrey Institute, Northwestern University, Evanston, IL 60208, USA \\ ${ }^{\mathrm{h}}$ Chemistry of Life Processes Institute, Northwestern University, Evanston, IL 60208, USA \\ ${ }^{\ddagger}$ These authors contributed equally to this work.
}

\section{Corresponding Authors}

*v-dravid@northwestern.edu

*nathan.gianneschi@northwestern.edu

*wdichtel@northwestern.edu

\section{Supplementary Information}

Correspondence Address
Professors William R. Dichtel and Nathan
Department of Chemistry
Northwestern University
2145 Sheridan Road
Evanston, IL 60208 USA

Tel: (+1) 847-467-6031, (+1) 847-491-2105

Email: wdichtel@northwestern.edu,nathan.gianneschi@northwestern.edu,

Professor Nathan C. Gianneschi and Vinayak P. Dravid

Department of Materials Science and Engineering

Northwestern University

2220 Campus Drive

Evanston, IL 60208 USA

Tel: (+1) 847-491-2105, (+1) 847-467-1363

Email: nathan.gianneschi@northwestern.edu,v-dravid@northwestern.edu 


\section{Table of Contents}

I. Materials and Methods S-3

II. Experimental Procedures $\quad$ S-5

III. Optical Images of Graphene Coated TEM Grids $\quad$ S-7

IV. Transmission Electron Microscopy Images $\quad$ S-8

V. MATLAB Script: Selecting a ROI/Pixel Frame S-15

VI. MATLAB Script: Selecting Mapping Script Accuracy $\quad$ S-19

VII. MATLAB Script: Selecting Phi Degree Binning S-23

VIII. MATLAB Script: Domain Mapping $\quad$ S-24

IX. Analysis of COF-5 Grain Boundaries $\quad$ S-25

X. Mapping Other Boronate Ester-Linked COFs $\quad$ S-30

XI. Mapping Other Materials $\quad$ S-33

XII. Grazing-Incidence Wide-Angle X-Ray Scattering S-34

$\begin{array}{ll}\text { XIII. Atomic Force Microscopy } & \text { S-38 }\end{array}$

XIV. References $\quad$ S-40 


\section{Materials and Methods}

$\underline{\text { Materials }}$

2,7-pyrenebis(boronic acid) ${ }^{1}$ and 4,4'-diphenylbutadiynebis(boronic acid) ${ }^{2}$ were prepared according to literature conditions. NMR data is consistent with those previously reported. Reagents were purchased in reagent grade from commercial suppliers and used without further purification, unless otherwise described. Solvents were obtained from commercial sources and used without further purification.

PELCO $^{\circledR}$ Single Layer Graphene TEM Support Films on Lacey Carbon, 300 Mesh Copper Grids (Ted Pella, Redding, CA) were used for all TEM experiments. The thickness for a single layer of graphene is approximately $0.35 \mathrm{~nm}$ with a transparency on the order of $96.4 \%$.

Monolayer graphene $\mathrm{SiO}_{2} / \mathrm{Si}(10 \mathrm{~mm} \times 10 \mathrm{~mm})$ substrates (University Wafer) were used for all GI-WAXS and AFM experiments.

Optical Images of Single Layer Graphene Coated TEM Grids and $\mathrm{SiO}_{2}$ Substrates

Optical images of the TEM grids were obtained using a trinocular biological compound microscope (T490B-PL, AmScope) equipped with an 18 MP camera.

Grazing-Incidence Wide-Angle X-Ray Diffraction

Grazing-incidence wide-angle X-ray scattering (GI-WAXS) measurements were performed at Sector 8-ID-E (beam energy $=10.92 \mathrm{keV}$ ) of the Advanced Photon Source at Argonne National Laboratory. Samples were placed under vacuum and aligned to provide an incident angle of $\sim 0.14^{\circ}$. Frames were collected for an amount of time such that the maximum pixel intensity did not exceed 80\%. Diffraction patterns were collected using a Pilatus 2D detector. All radially integrated patterns were found to agree with previously reported diffraction patterns.

\section{Atomic Force Microscopy}

Atomic force microscopy (AFM) images were collected using a Bruker Dimension Fastscan AFM in tapping mode.

Transmission Electron Microscopy

TEM was performed using a JEOL (JEOL USA, Inc., Peabody, MA) ARM300F GrandARM TEM operating at $300 \mathrm{keV}$ equipped with a Gatan (Gatan, Inc., Pleasanton, CA) K3-IS "direct electron" detector (FEG Emission: $15 \mu \mathrm{A}$, spot size 5, $150 \mu \mathrm{m}$ CL aperture). The ARM300F was aligned for low-dose imaging, measuring the dose rate on the $\mathrm{K} 3$ detector through vacuum (no grid inserted). The dose rate was measured to be 2.1-19.6 $\mathrm{e}^{-} \AA^{-2} \mathrm{~s}^{-1}$ (5760 x 4092 pixels) with image exposure times of 0.2-1 s (0.4-19.5 $\mathrm{e}^{-} \AA^{-2}$ cumulative dose per image).

For Figures S2-S3 TEM was performed using a JEOL (JEOL USA, Inc., Peabody, MA) ARM200CF Aberration-Corrected STEM/TEM operated at $200 \mathrm{keV}$ equipped with a Gatan (Gatan, Inc., Pleasanton, CA) K2 “direct electron" detector (FEG Emission: $5 \mu$ A, spot size 3, 150 $\mu \mathrm{m} C L$ aperture). The ARM200CF was aligned for low-dose imaging, measuring the dose rate on the K2 detector through vacuum (no grid inserted). The dose rate was measured to be 
3.0-3.7 $\mathrm{e}^{-} \AA^{-2} \mathrm{~s}^{-1}(3710 \times 3838$ pixels $)$ with image exposure times of $1 \mathrm{~s}\left(3.0-3.6 \mathrm{e}^{-} \AA^{-2}\right.$ cumulative dose per image).

All image acquisition was done using the Gatan Microscopy Suite (GMS), Digital Micrograph (Gatan, Inc., Pleasanton, CA).

Geometry Optimization

Simulations of molecular defects in 2D COF monolayers were carried out in Materials Studio (ver.5.0). ${ }^{3}$ First, the unit cell was constructed piecewise in a highly symmetric $P 6 / \mathrm{mmm}$ unit cell with the $a=b$ lattice parameter set to be approximately the distance between two COF vertices. The $c$ parameter was set to be $3.5 \AA$, which is the interlayer spacing of graphene. We then used a Forcite geometry optimization routine with a universal forcefield to optimize the unit cell size with convergence tolerances of: Energy $=10^{-3} \mathrm{kcal} \mathrm{mol}^{-1}$ and Force $=0.5 \mathrm{kcal} \mathrm{mol}^{-1} \AA^{-1}$. This routine produced the COF-5 unit cell we have observed in previous reports. ${ }^{4}$ We then created a supercell monolayer of this structure (Figure 5C). Next, we removed all crystallographic constraints to produce a large-area COF-5 sheet. Then, we selected an unreacted boronic acid at the edge of a COF crystal and installed a hemiboronate-ester at that position. From that position we constructed a second pristine COF sheet from that defect site of approximately the same size as the first. Once a crude version of the defective $\mathrm{COF}$ sheet was produced, we then conducted a geometry optimization routine using Forcite with the convergence tolerances of $10^{-5} \mathrm{kcal} \mathrm{mol}^{-1}$ and $10^{-3} \mathrm{kcal}$ $\mathrm{mol}^{-1} \AA^{-1}$ and displacement of $10^{-5} \AA$. This produced the structure observed in Figures 5C-5D that showed a 12-degree offset between domains. Importantly, each individual COF domain, outside of the defective boundary, relaxed nicely to the expected COF structure. 


\section{Experimental Procedures}

Synthesis of Colloidal COF Films and TEM Sample Preparation

2,3,6,7,10,11-Hexahydroxytriphenylene (HHTP, $1 \mathrm{mM}$ ) and the appropriate corresponding boronic acid (BA, $1.5 \mathrm{mM}$ ) were dissolved in a mixture of $\mathrm{CH}_{3} \mathrm{CN}$ :1,4-dioxane:mesitylene $(80 / 16 / 4 \mathrm{v} / \mathrm{v} / \mathrm{v} ; 20 \mathrm{~mL})$ and sonicated for $5 \mathrm{~min}$. The solution was passed through Fisherbrand qualitative grade plain filter paper (diameter: $5.5 \mathrm{~cm}, \mathrm{P} 4$ grade) via vacuum filtration to remove insoluble particulates.

All COF films (COF-5, TP-COF, DPB-COF, and COF-10) were prepared via solvothermal growth. For each system, $20 \mathrm{~mL}$ of monomer solution was placed in a $20 \mathrm{~mL}$ scintillation vial. A $\mathrm{PELCO}^{\odot}$ single layer graphene (lacey-carbon, $\mathrm{Cu}, 300$ mesh) TEM grid (Ted Pella, Redding, CA) and a $10 \mathrm{~mm} \times 10 \mathrm{~mm}$ monolayer graphene $\mathrm{SiO}_{2} / \mathrm{Si}$ substrate (University Wafer) was placed in each vial. The vials were sealed and heated on a hotplate at $70{ }^{\circ} \mathrm{C}$ without stirring for $24 \mathrm{~h}$, which resulted in stable, colloidal suspensions.

The colloidal solutions were then decanted from the vials and the TEM grid and substrate were retrieved using tweezers. The TEM grids were immediately rinsed with fresh $\mathrm{CH}_{3} \mathrm{CN}$ :1,4dioxane:mesitylene $(80 / 16 / 4 \mathrm{v} / \mathrm{v} / \mathrm{v} ; 20 \mathrm{~mL})$ solvent and allowed to dry open to air. The TEM grids were then stored in a standard grid box inside of a desiccator prior to imaging.

\section{Mapping Script Information}

Scripts were written using MATLAB version 2019b. Image and Metadata importing was done using a modified version of a file publicly available on MATLAB's Central File Exchange. ${ }^{5}$

The user first selects a region of interest (ROI) size in pixels, known as the pixel frame. This number is the side of a square in pixels. A smaller ROI has advantages in terms of accuracy and amount of data while a larger ROI has improved signal to noise. For this work we selected a ROI size that houses at least twenty repeat units. Next, a spatial accuracy is selected, corresponding to the size of the square in the final mapped image. The accuracy determines how much the ROIs overlap as they raster across the image. The entire image is then subjected to a two-dimensional (2D) Fourier-transform, which is then integrated in $\mathrm{Q}_{\mathrm{xy}}$ space to find the location of the primary repeat spacing in the image. This is then transformed to a real space number, which is used to determine appropriate bandpass filtering in later steps. Alternatively, the user can specify the repeat unit spacing in real space, if that spacing is known a priori. Next, the ROI is scanned across the entire image, generating FFTs at each ROI and radially integrating the intensities (phi integration). The location of the maximum intensity spot in each FFT and corresponding phi degree are then extracted. If the phi degree of the maximum intensity spot in the FFT differs by greater than the phi degree binning number (imposed by the user in the script), that corresponds to a new domain in the image. Each color in the domain orientation map corresponds to a specific lattice orientation as defined by the phi angle measured from the FFTs.

The identification of the brightest spot of a region of interest's (ROI) FFT was accomplished by the following workflow. 1) First the FFT was masked (five pixel circle mask) to block intensities of the center spot (i.e. these intensities were reduced to zero). 2) The masked FFT was bandpass filtered to select for the FFT intensity related to the 100 feature (i.e. spacings related to the 100 
spacing $\pm 5 \AA$ ). 3) Due to the two-fold symmetry of FFT patterns, we then summed each half of the radial intensities (i.e. $0-180^{\circ}$ and $180-360^{\circ}$, taking into account the rotational offset). 4) This intensity was then radially integrated to produce a $1 \mathrm{D}$ plot of intensity versus phi angle from 0 $180^{\circ}$. 5) The maximum intensity was then identified from this plot.

Unless otherwise noted, the mapping script accuracy, pixel frame, and phi degree binning used to map the images in this manuscript were $3 \mathrm{~nm}, 500$ pixel frames, and $5^{\circ}$, respectively.

All scripts are available on GitHub. 


\section{Optical Images of Graphene Coated TEM Grids}
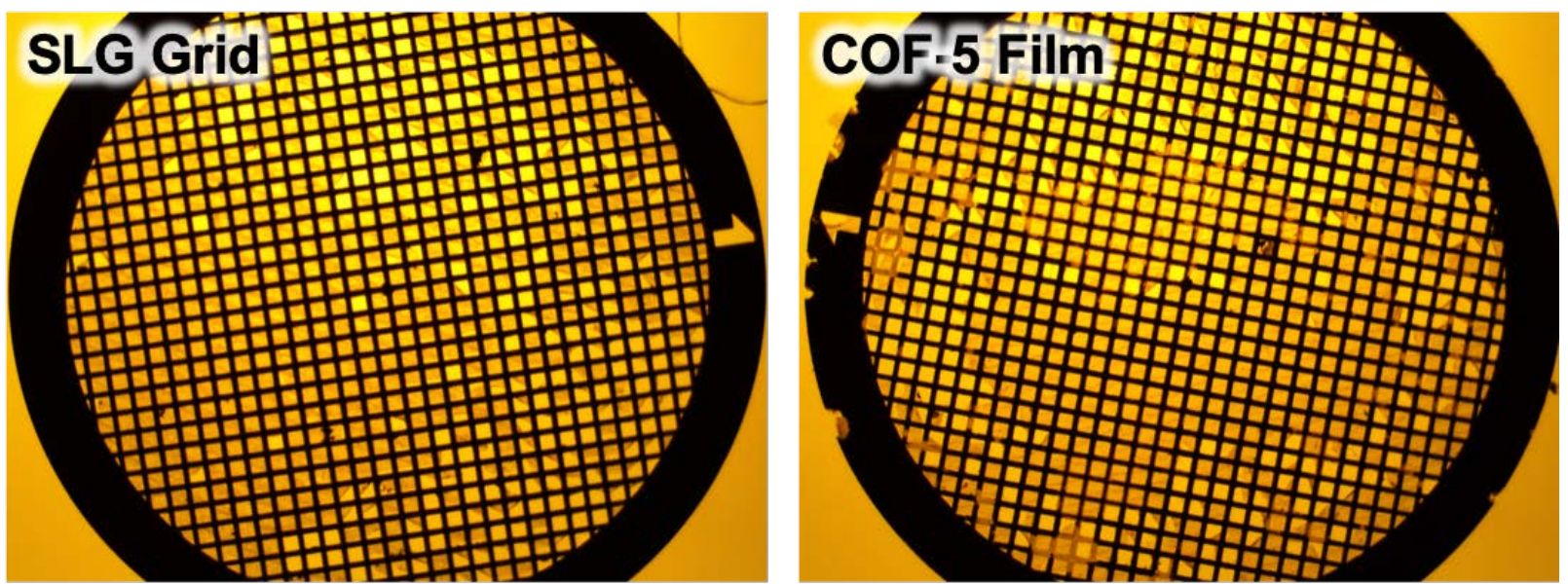

SLG Grid

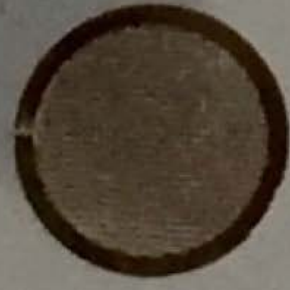

COF -5 Film

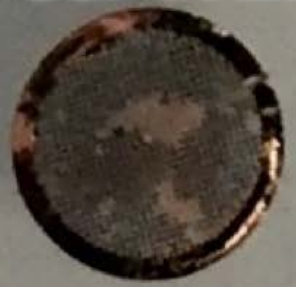

Figure S1. Optical images of the single layer graphene coated TEM grids before (left) and after (right) COF-5 film growth. Image of the grid was taken after extensive handling of the grid which caused several of the tears in the film. 
IV. Transmission Electron Microscopy Images
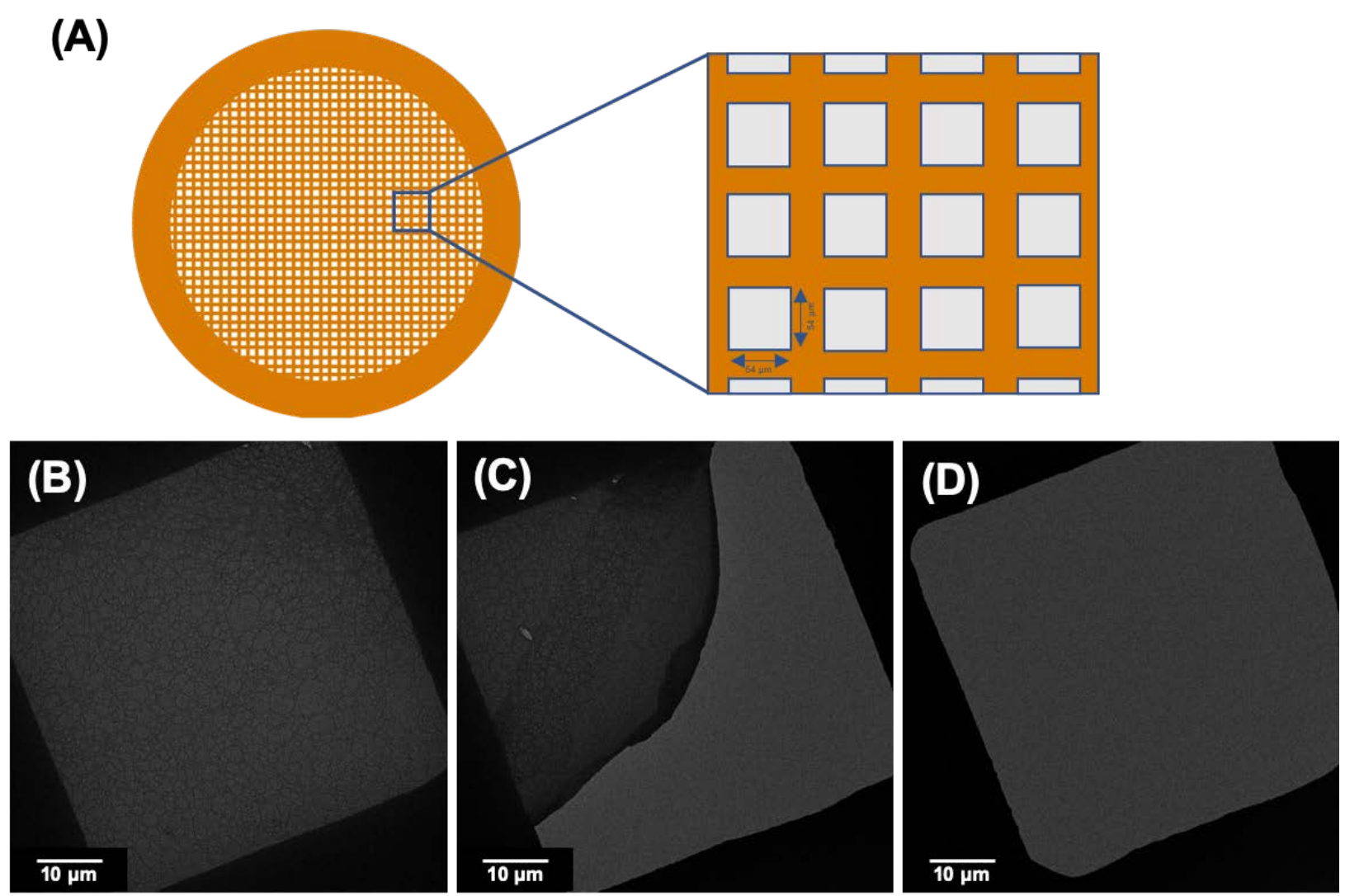

Figure S2. (A) Schematic showing a standard TEM grid with $54 \mu \mathrm{m}$ x $54 \mu \mathrm{m}$ square windows. Low magnification TEM images showing different possible conditions of the TEM grid bars after film synthesis: (B) fully coated with lacey carbon and COF film, (C) partially covered with the COF film rolled towards the upper right corner, and (D) completely empty.
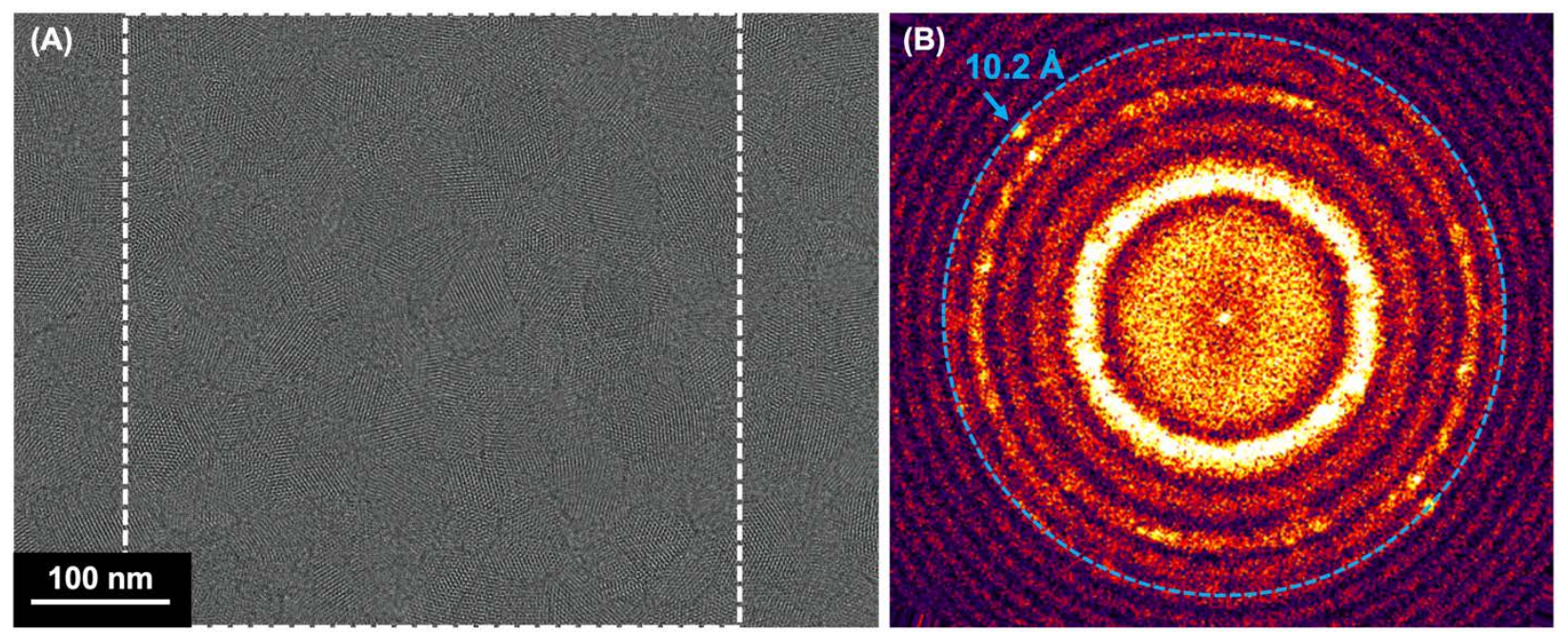

Figure S3. TEM Image Resolution. (A) TEM image of COF-5 film. (B) FFT of boxed region in (A) showing rings due to the polycrystalline nature of the films. The blue circle marks the farthest observable spots in the FFT which is indicative of the image resolution. 

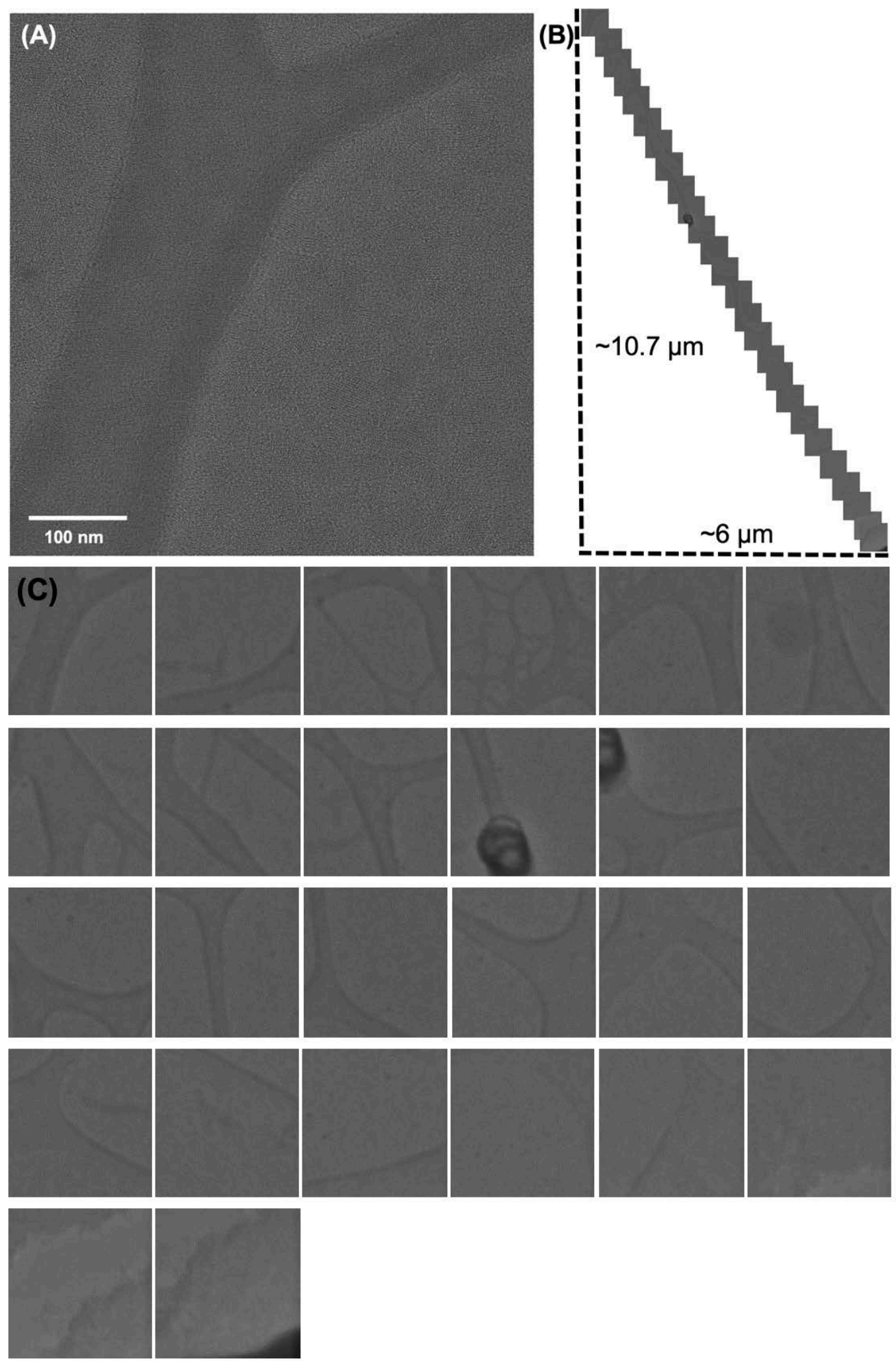

Figure S4. (A) Lattice resolution image of a COF-5 film. (B) 26 images taken at the same magnification moving by a set value within a single $54 \mu \mathrm{m} \times 54 \mu \mathrm{m}$ grid bar showing the extent of crystalline COF-5 film coverage. (C) TEM images shown in (B) prior to stitching them together, where each image is approximately $530 \mathrm{~nm} \times 550 \mathrm{~nm}$. 

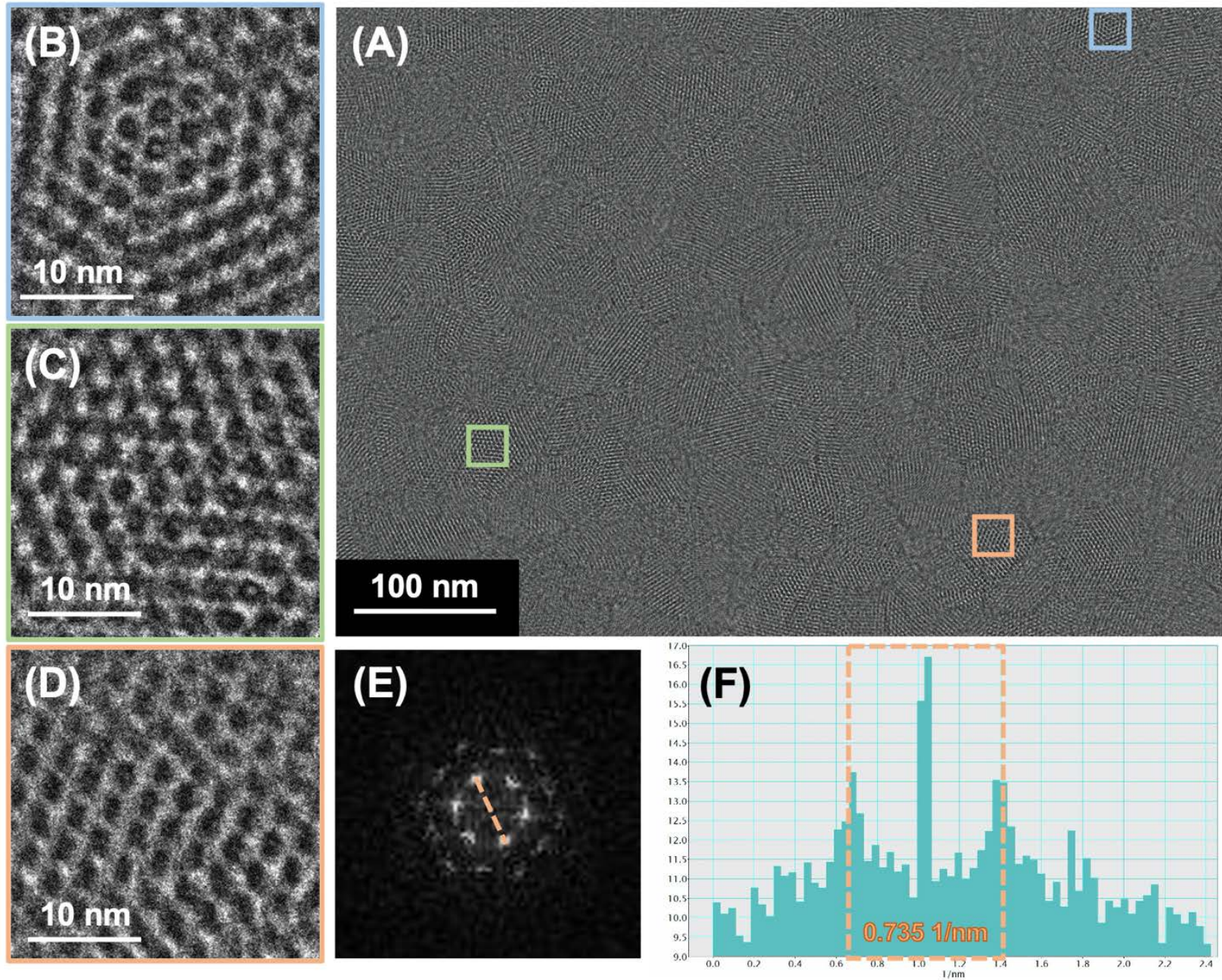

Figure S5. Observation of COF Pores. (A) HR-TEM image of COF-5 film. (B) Magnified images of blue boxed region, (C) green boxed region, and (D) orange boxed region showing COF pores. (E) FFT of orange boxed region showing a hexagonal pattern of spots. (F) Line scan of the FFT in (E) showing the distance between the spots in the hexagonal pattern. The spots are 0.735 $\mathrm{nm}^{-1}$ apart which corresponds to a spacing of $27 \AA$ and matches the theoretical size of a COF-5 pore. 

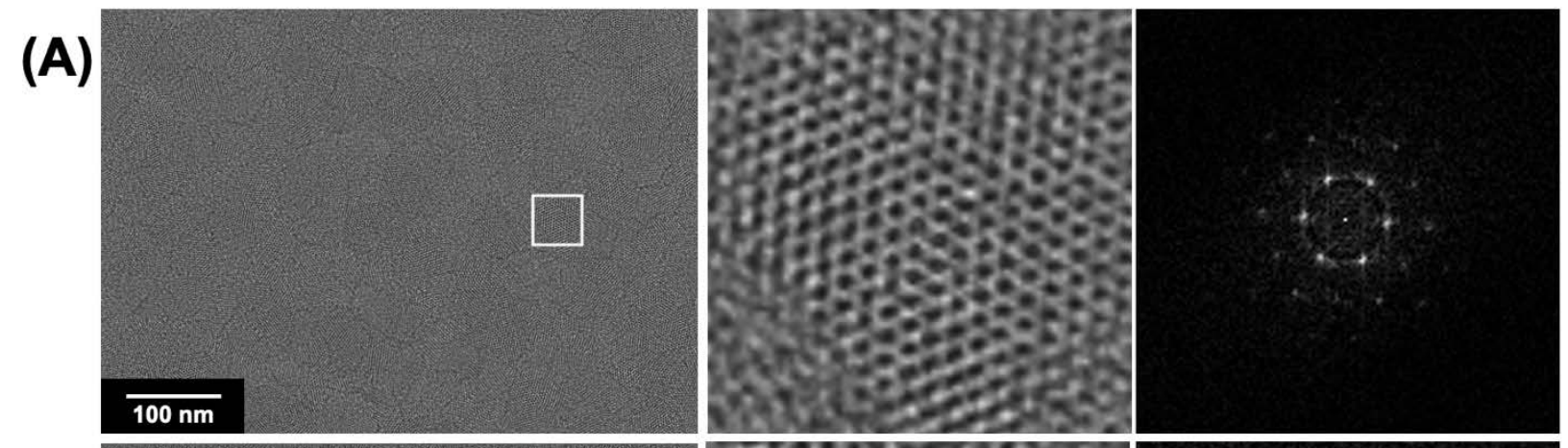

(B)
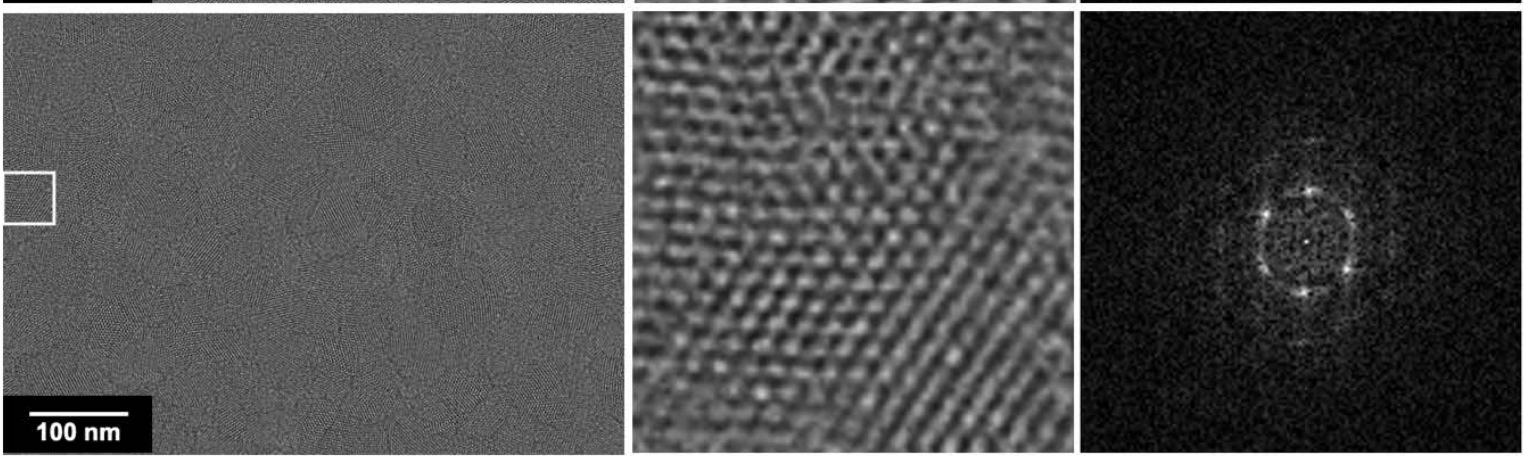

(C)
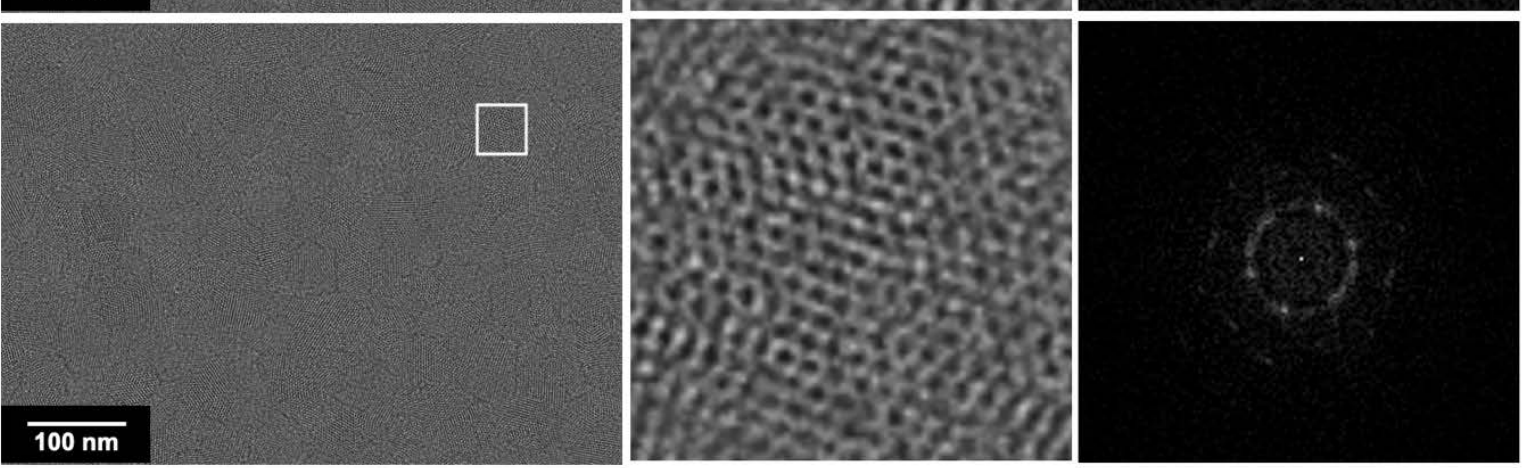

(D)
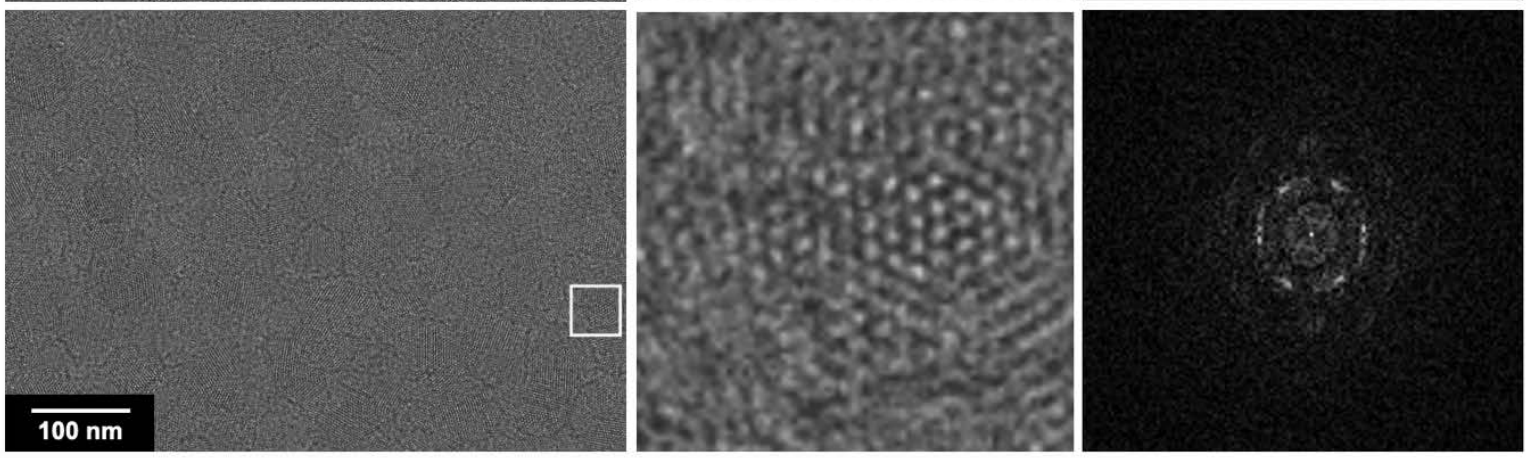

Figure S6. Additional TEM images of COF-5 film grown on single layer graphene TEM grids: Full HR-TEM image (left), magnified boxed region (middle), and FFT of boxed region (right). Dose Rate / Cumulative Dose: (A) $13.90 \mathrm{e}^{-} \AA^{-2} \mathrm{~s}^{-1} / 13.84 \mathrm{e}^{-} \AA^{-2}$, (B) $13.81 \mathrm{e}^{-} \AA^{-2} \mathrm{~s}^{-1} / 13.75 \mathrm{e}^{-} \AA^{-2}$, (C) $13.81 \mathrm{e}^{-} \AA^{-2} \mathrm{~s}^{-1} / 13.75 \mathrm{e}^{-} \AA^{-2}$, and (D) $12.64 \mathrm{e}^{-} \AA^{-2} \mathrm{~s}^{-1} / 12.59 \mathrm{e}^{-} \AA^{-2}$. 


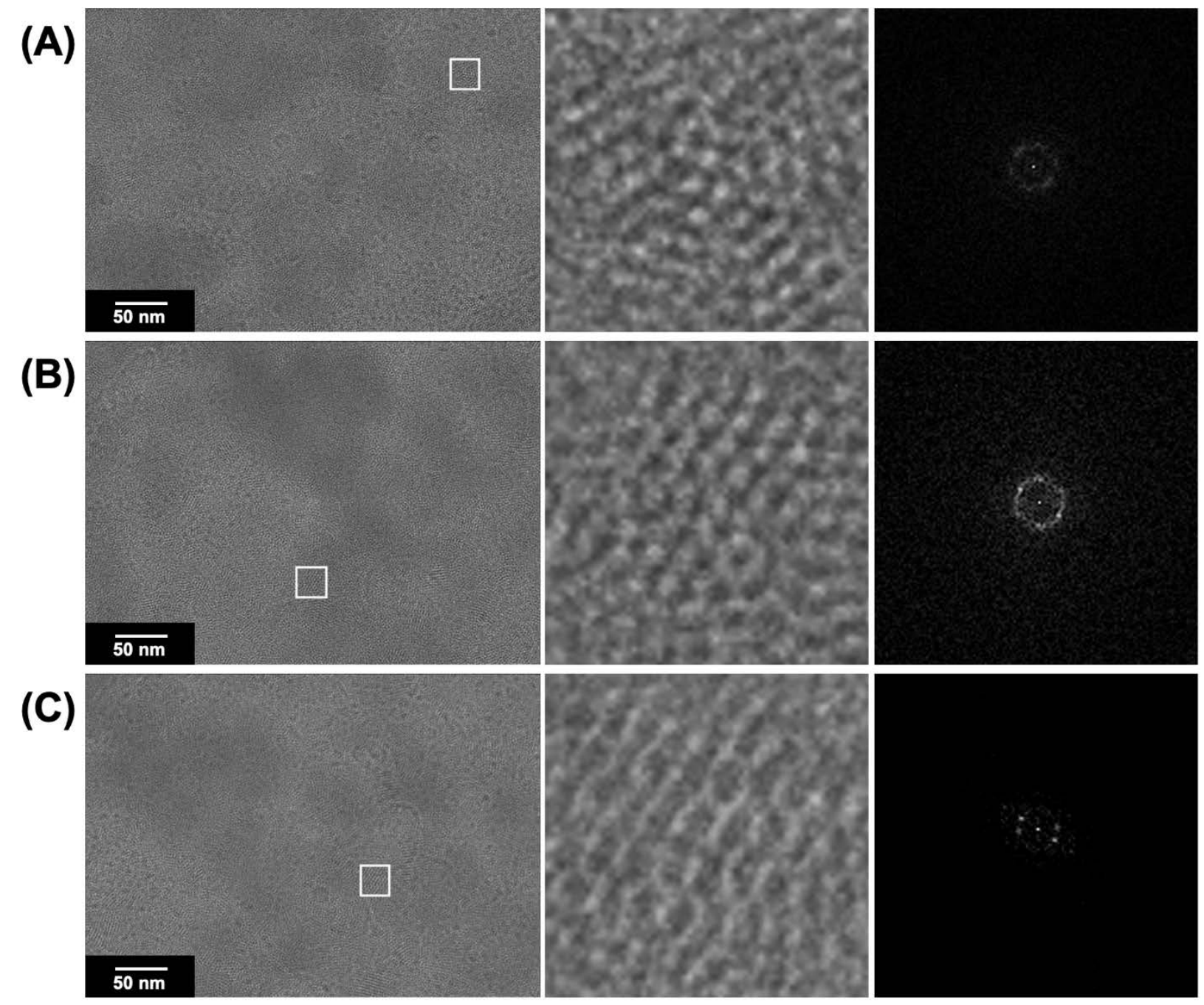

Figure S7. Additional TEM images of COF-10 film grown on single layer graphene TEM grids: Full HR-TEM image (left), magnified boxed region (middle), and FFT of boxed region (right). Dose Rate / Cumulative Dose: (A) $17.41 \mathrm{e}^{-} \AA^{-2} \mathrm{~s}^{-1} / 17.38 \mathrm{e}^{-} \AA^{-2}$, (B) $17.31 \mathrm{e}^{-} \AA^{-2} \mathrm{~s}^{-1} / 17.28 \mathrm{e}^{-} \AA^{-2}$, and (C) $17.35 \mathrm{e}^{-} \AA^{-2} \mathrm{~s}^{-1} / 17.32 \mathrm{e}^{-} \AA^{-2}$. 


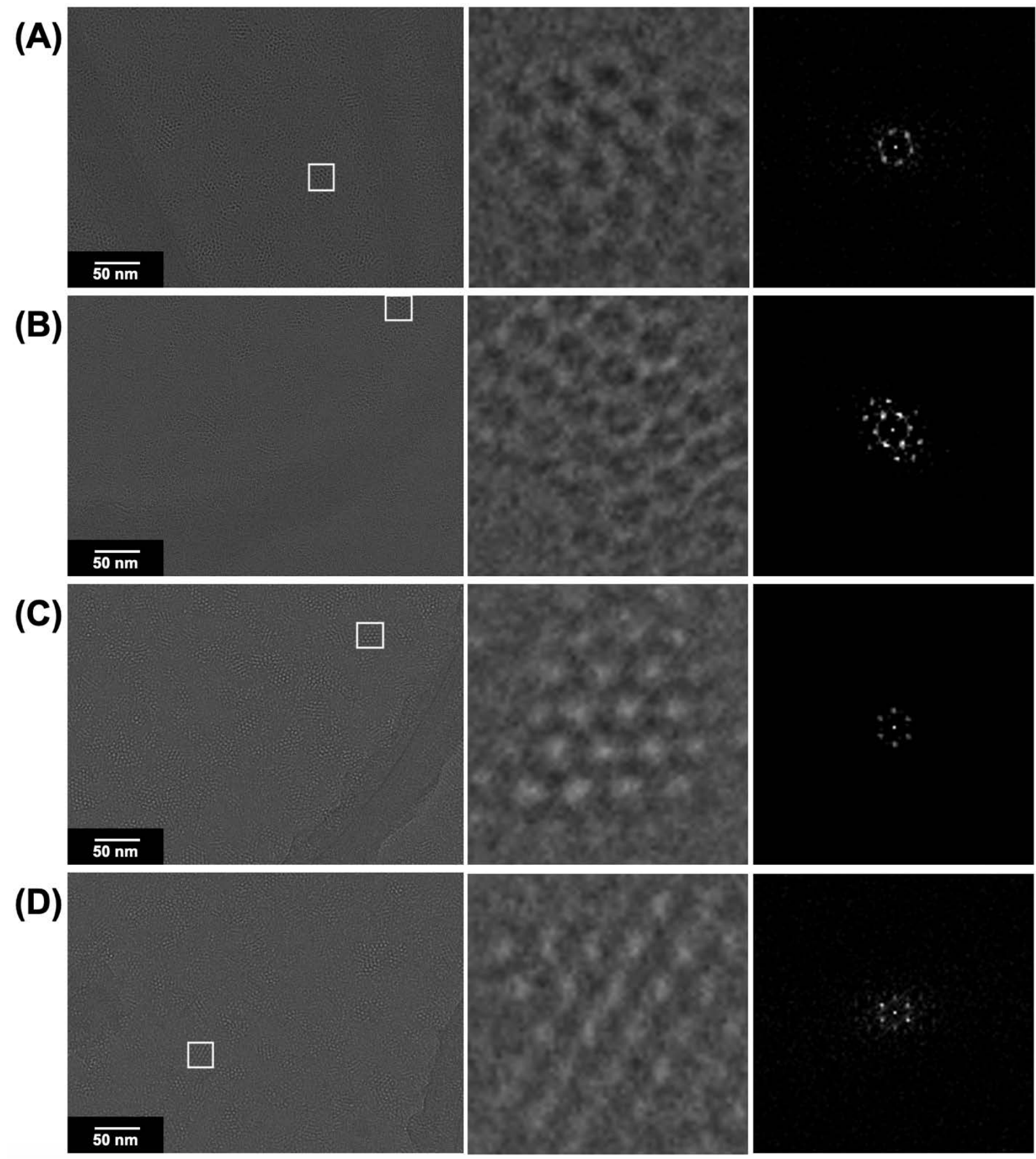

Figure S8. Additional TEM images of DPB-COF film grown on single layer graphene TEM grids: Full HR-TEM image (left), magnified boxed region (middle), and FFT of boxed region (right). Dose Rate / Cumulative Dose: (A) $9.60 \mathrm{e}^{-} \AA^{-2} \mathrm{~s}^{-1} / 3.73 \mathrm{e}^{-} \AA^{-2}$, (B) $10.77 \mathrm{e}^{-} \AA^{-2} \mathrm{~s}^{-1} / 4.19 \mathrm{e}^{-} \AA^{-2}$, (C) $16.49 \mathrm{e}^{-} \AA^{-2} \mathrm{~s}^{-1} / 6.41 \mathrm{e}^{-} \AA^{-2}$, and (D) $16.43 \mathrm{e}^{-} \AA^{-2} \mathrm{~s}^{-1} / 6.39 \mathrm{e}^{-} \AA^{-2}$. 


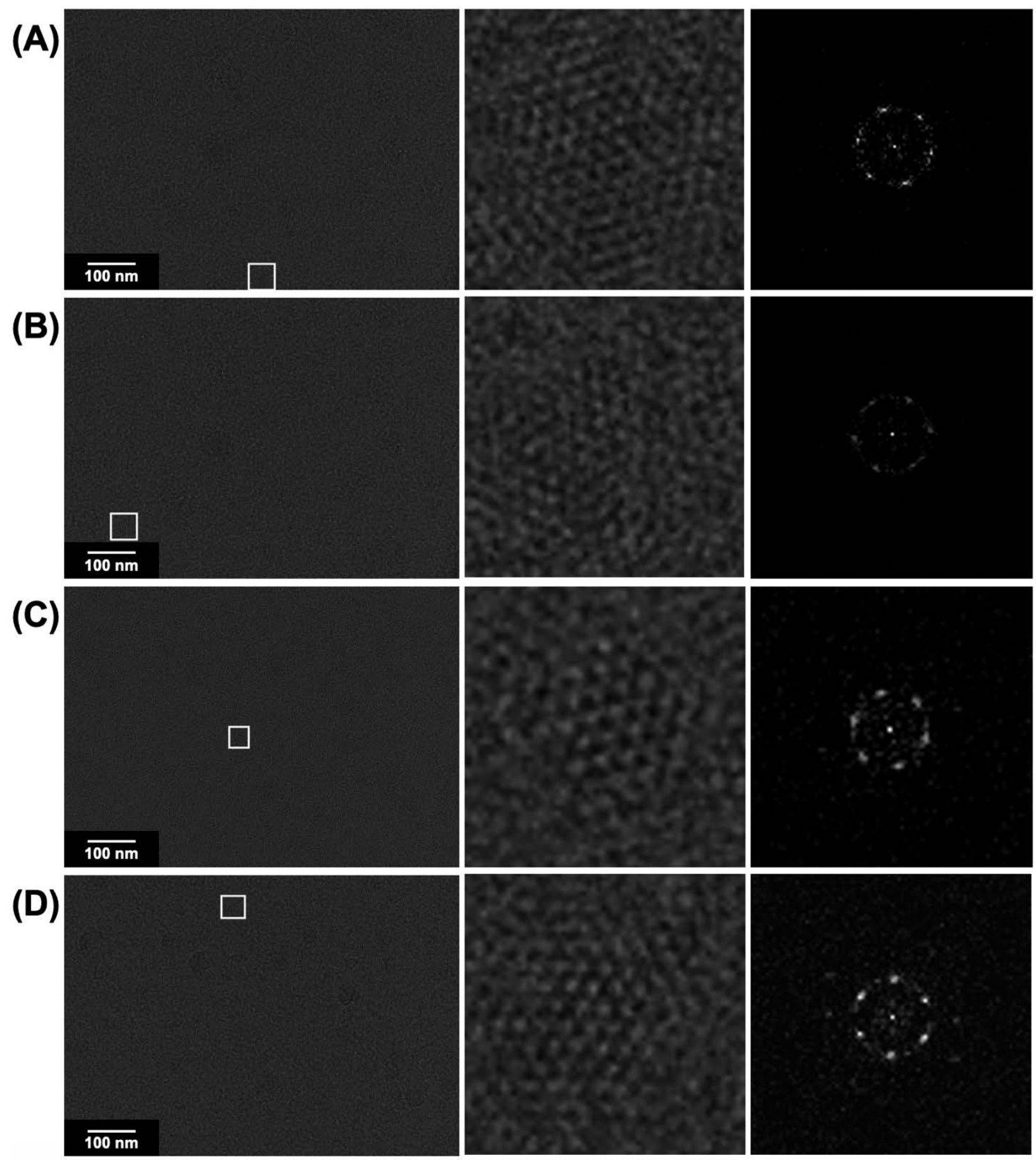

Figure S9. Additional TEM images of TP-COF film grown on single layer graphene TEM grids: Full HR-TEM image (left), magnified boxed region (middle), and FFT of boxed region (right). Dose Rate / Cumulative Dose: (A) $2.11 \mathrm{e}^{-} \AA^{-2} \mathrm{~s}^{-1} / 0.40 \mathrm{e}^{-} \AA^{-2}$, (B) $2.11 \mathrm{e}^{-} \AA^{-2} \mathrm{~s}^{-1} / 0.41 \mathrm{e}^{-} \AA^{-2}$, (C) $2.12 \mathrm{e}^{-} \AA^{-2} \mathrm{~s}^{-1} / 0.41 \mathrm{e}^{-} \AA^{-2}$, and (D) $2.15 \mathrm{e}^{-} \AA^{-2} \mathrm{~s}^{-1} / 0.41 \mathrm{e}^{-} \AA^{-2}$. 
V. MATLAB Script: Selecting a ROI/Pixel Frame

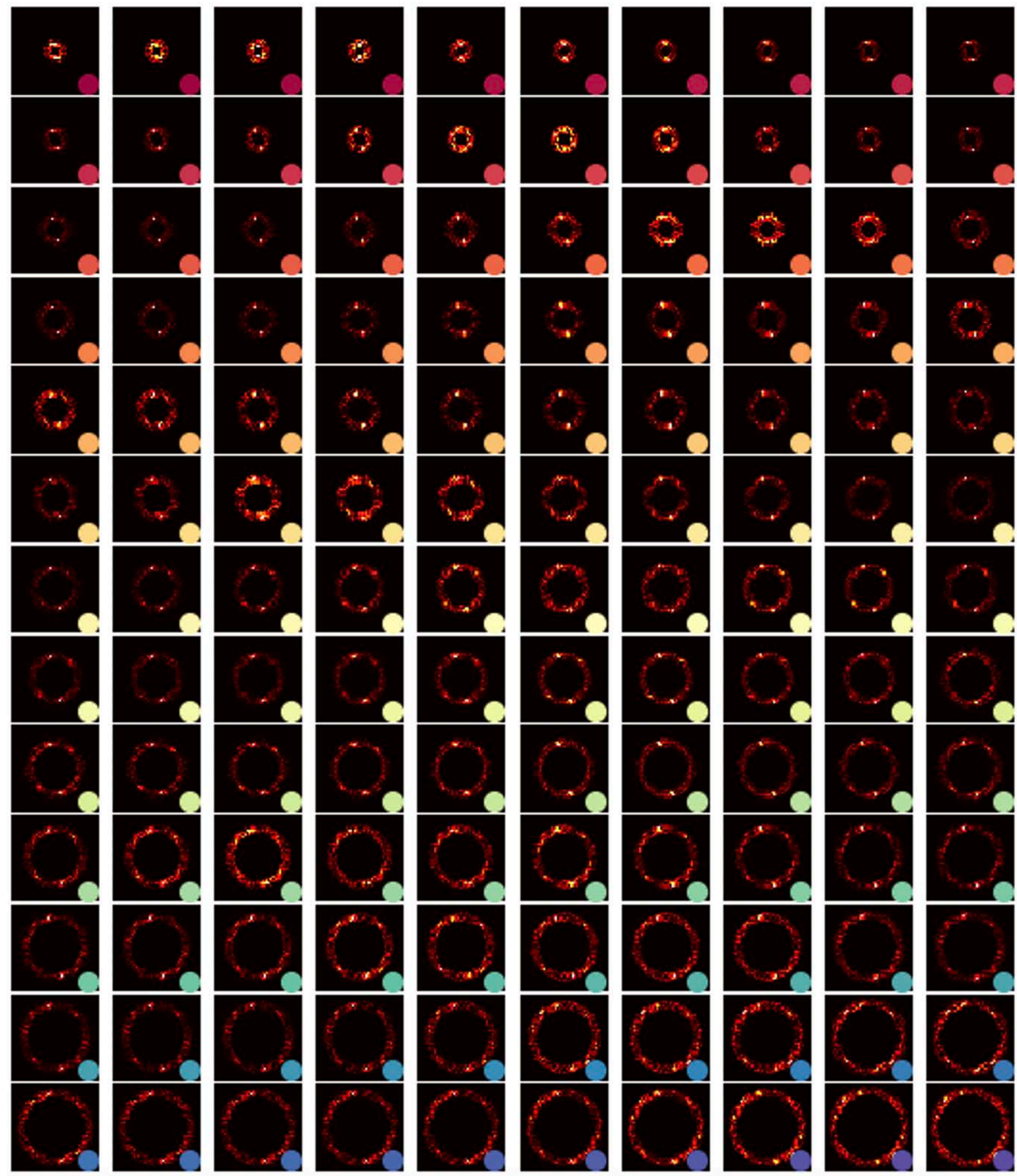

Figure S10. 2D Fourier transforms of the COF-5 film HR-TEM image shown in Figure 2A with ROI sizes between 300 (red) and 500 (purple) pixels. The images are bandpass filtered using the MATLAB mapping script previously described. Signal to noise ratios are then extracted to select the optimal ROI size for the image. 
(A)

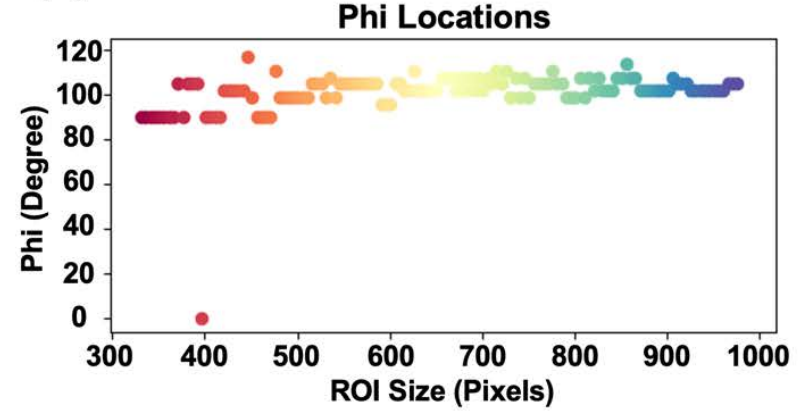

(C)

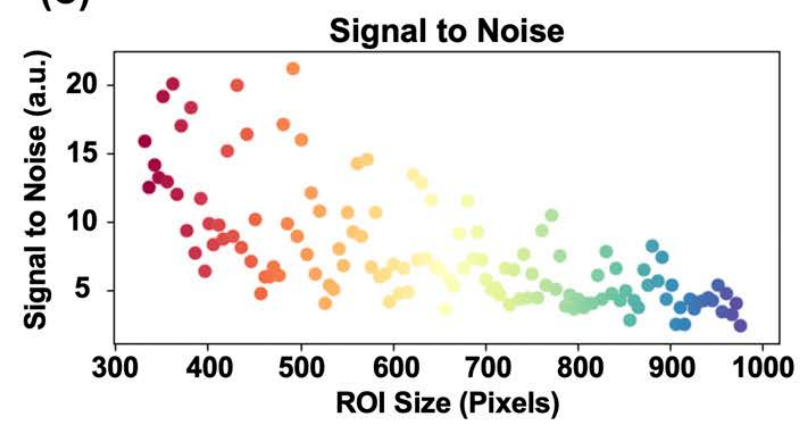

(B)

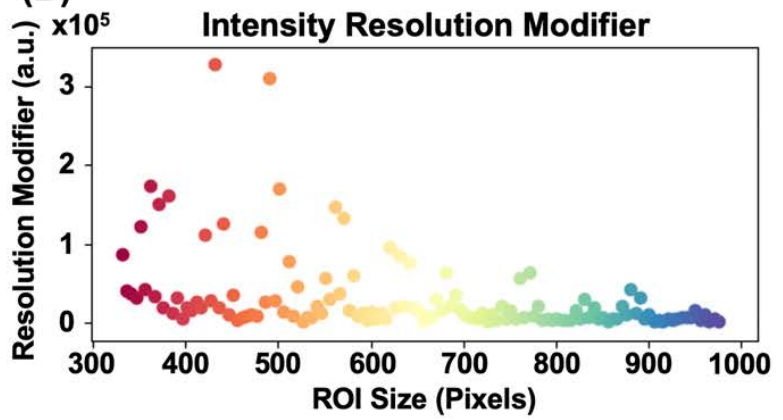

(D)

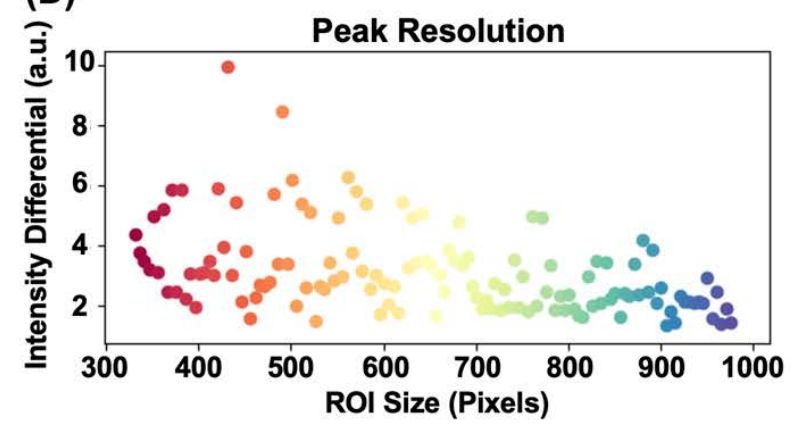

Figure S11. (A) Location of the most intense phi spot as a function of ROI size. At small ROIs, the script is unable to precisely assign the phi value which is later used to construct the domain map because of the limited number of pixels, and therefore limited phi accuracy, of the 2D Fourier transform. (B) The product of (C) and (D) versus pixel size which, in conjunction with the phi accuracy, is used to assign the optimal ROI size for a given image. (C) Signal to noise versus ROI size is computed by assessing the maximum intensity versus the variance in binned phi intensity. (D) Peak accuracy versus ROI size is determined by the ratio of the local maximum intensities, which yields a higher value when a single set of FFT spots are observed. 

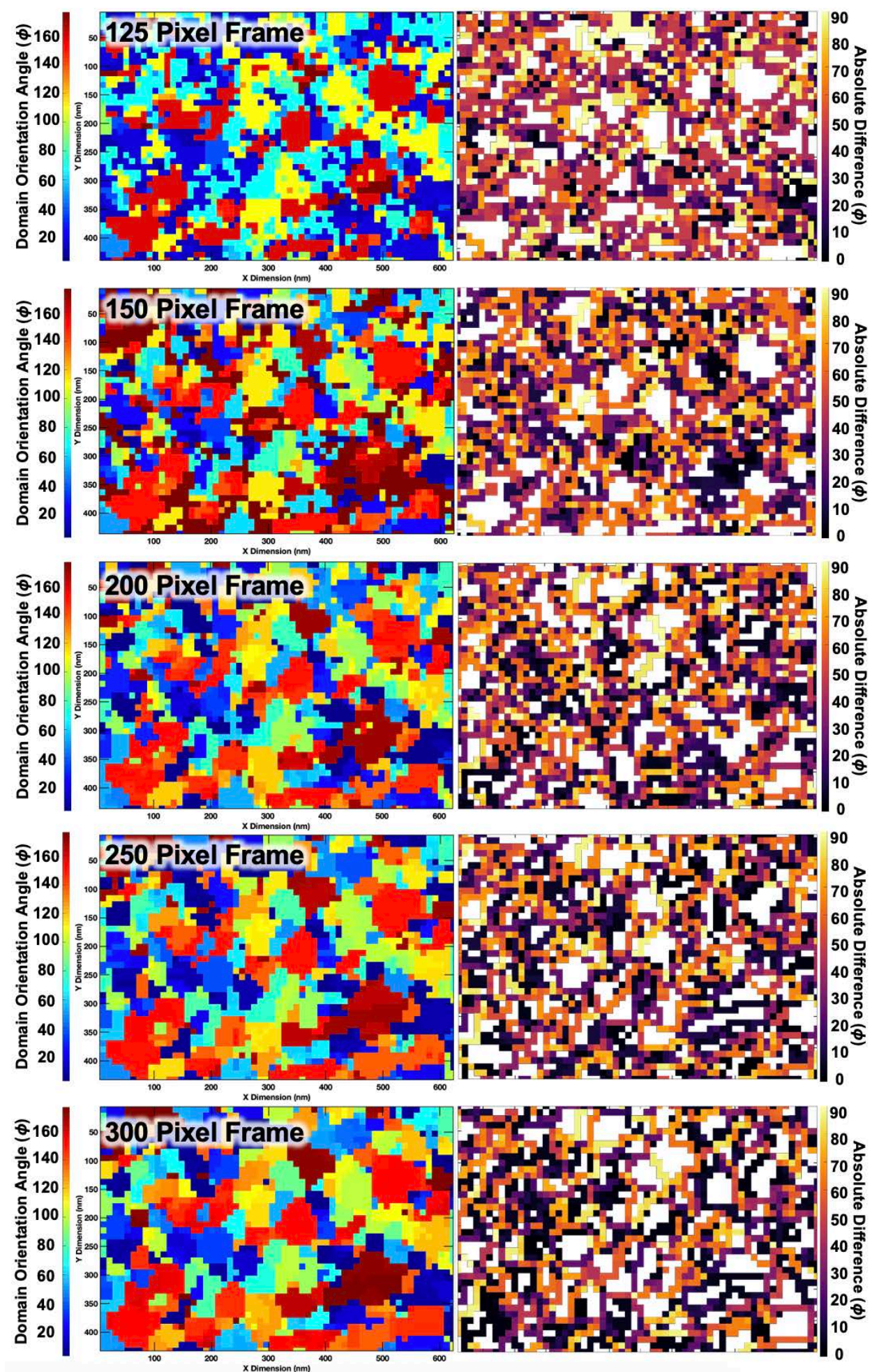

Figure S12. Selection of mapping pixel frame for the COF-5 film HR-TEM image shown in Figure 2A. Domain map (left) and corresponding grain boundary map (right) for pixel frames ranging from 125-300 ( $3^{\circ}$ phi binning used). 

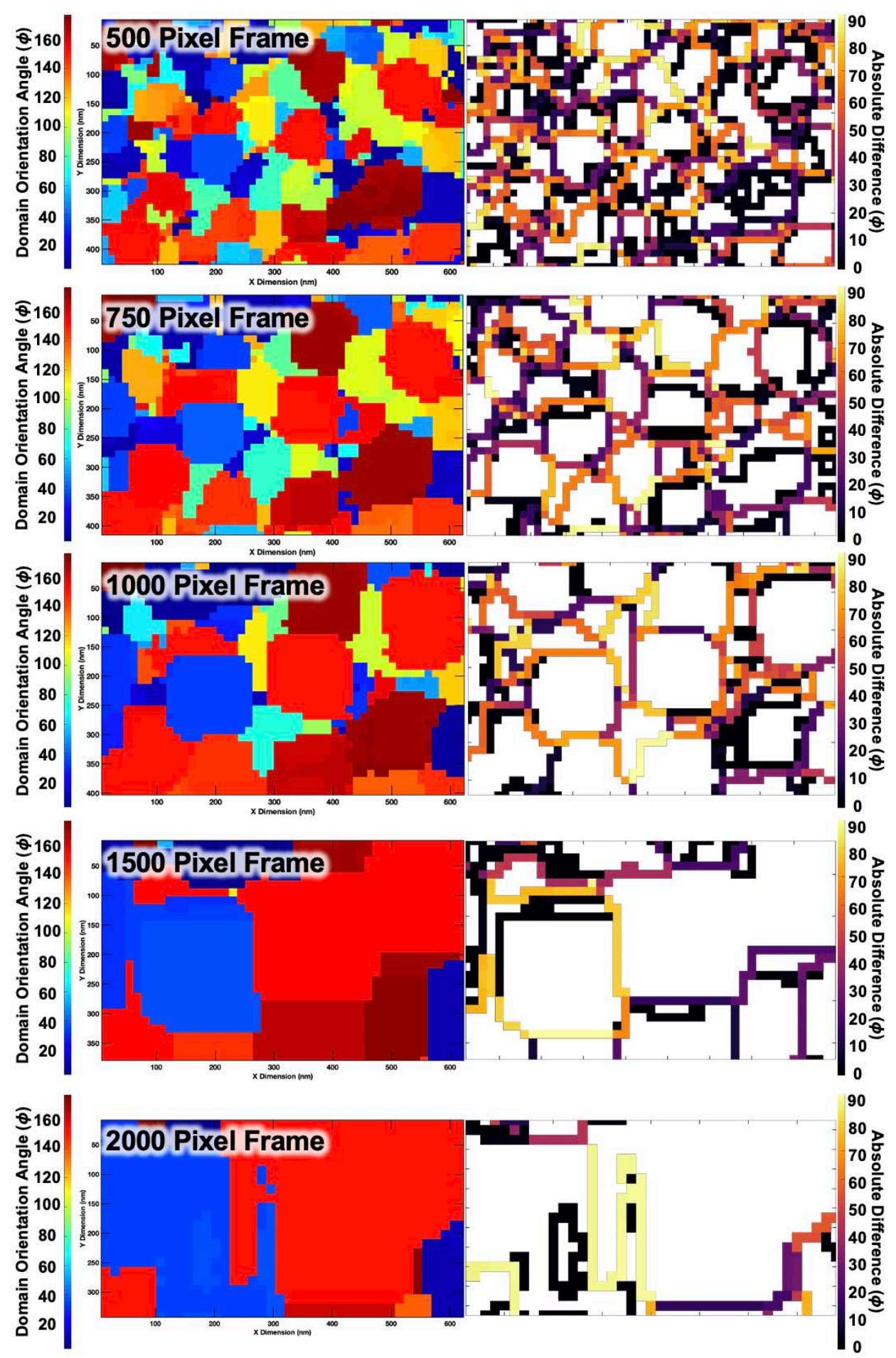

Figure S13. Selection of mapping pixel frame for the COF-5 film HR-TEM image shown in Figure $2 \mathrm{~A}$. Domain map (left) and corresponding grain boundary map (right) for pixel frames ranging from 500-2000 ( $3^{\circ}$ phi binning used). 
VI. MATLAB Script: Selecting Mapping Accuracy

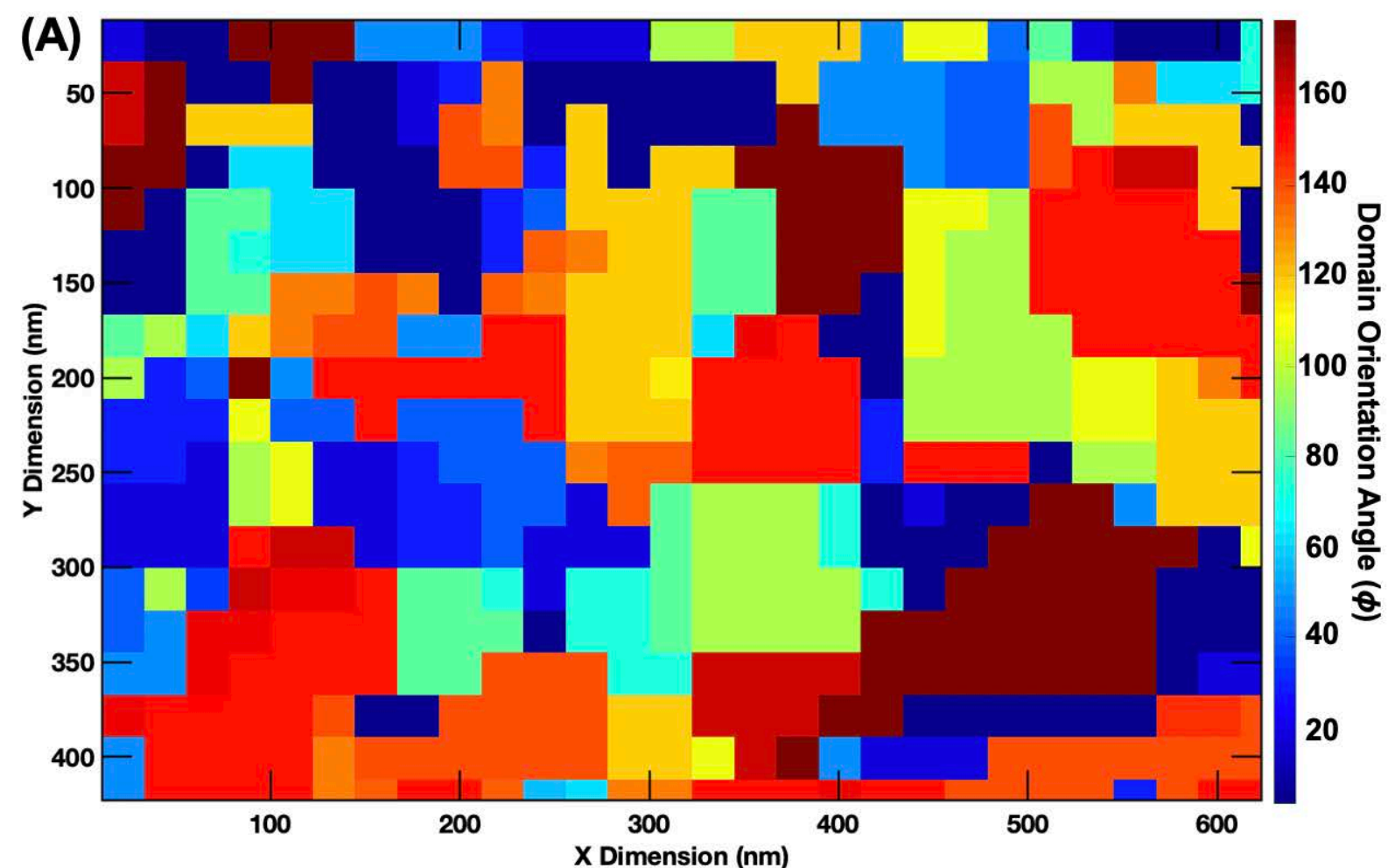

(B)

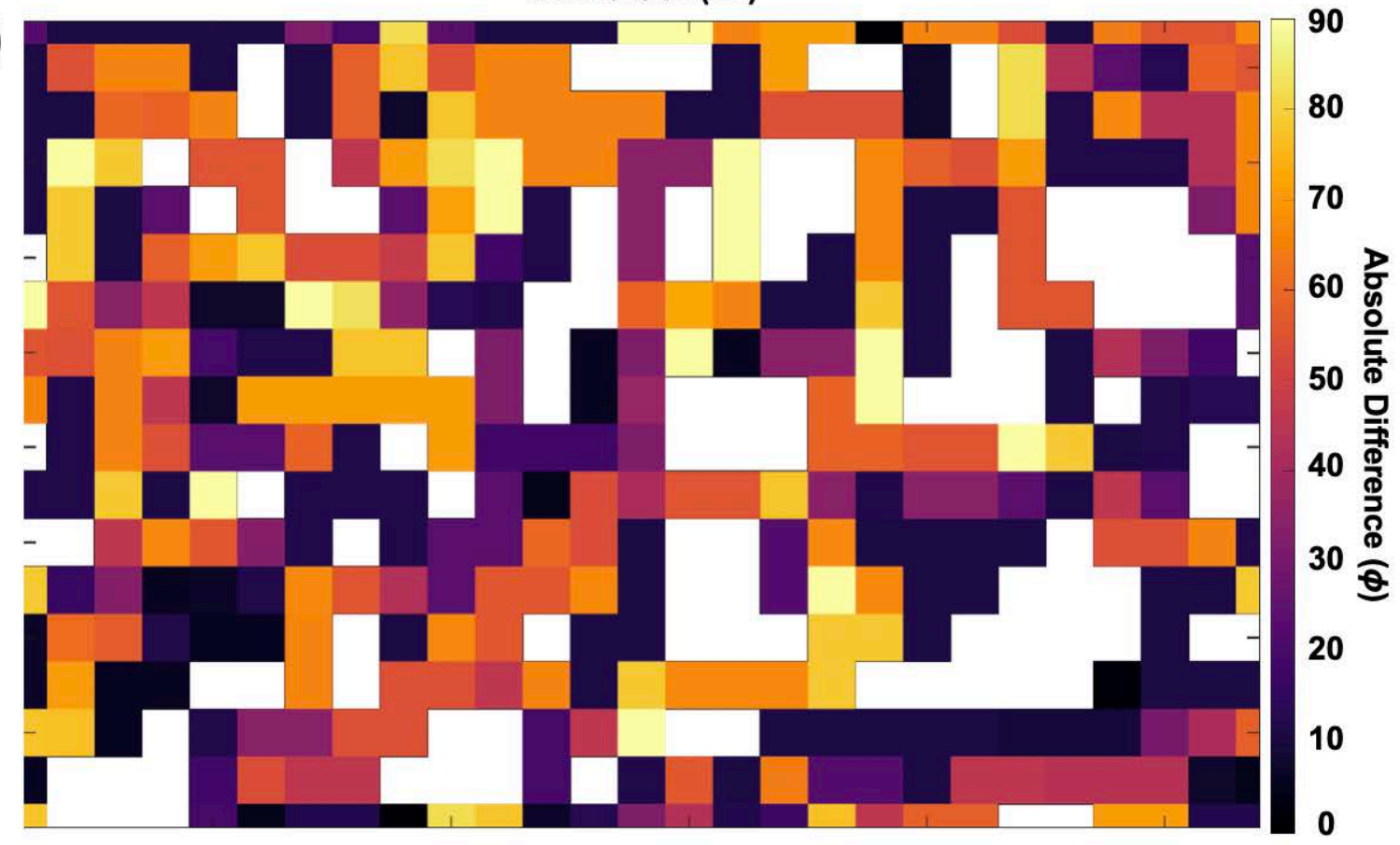

Figure S14. Selection of mapping spatial accuracy for the COF-5 film HR-TEM image shown Figure 2A. (A) Domain map using a $20 \mathrm{~nm}$ accuracy. (B) Grain boundary map using a $20 \mathrm{~nm}$ accuracy. 


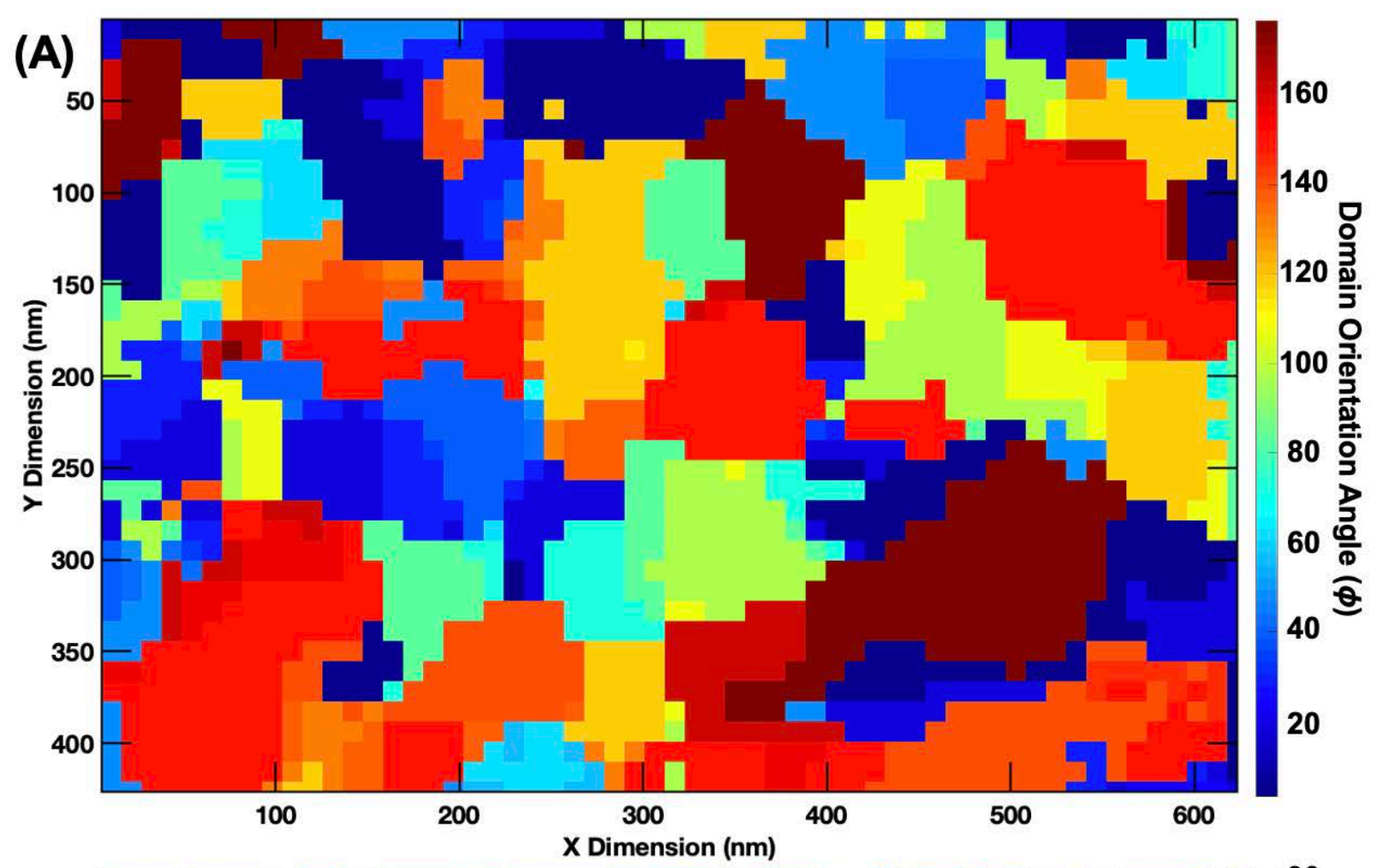

(B)

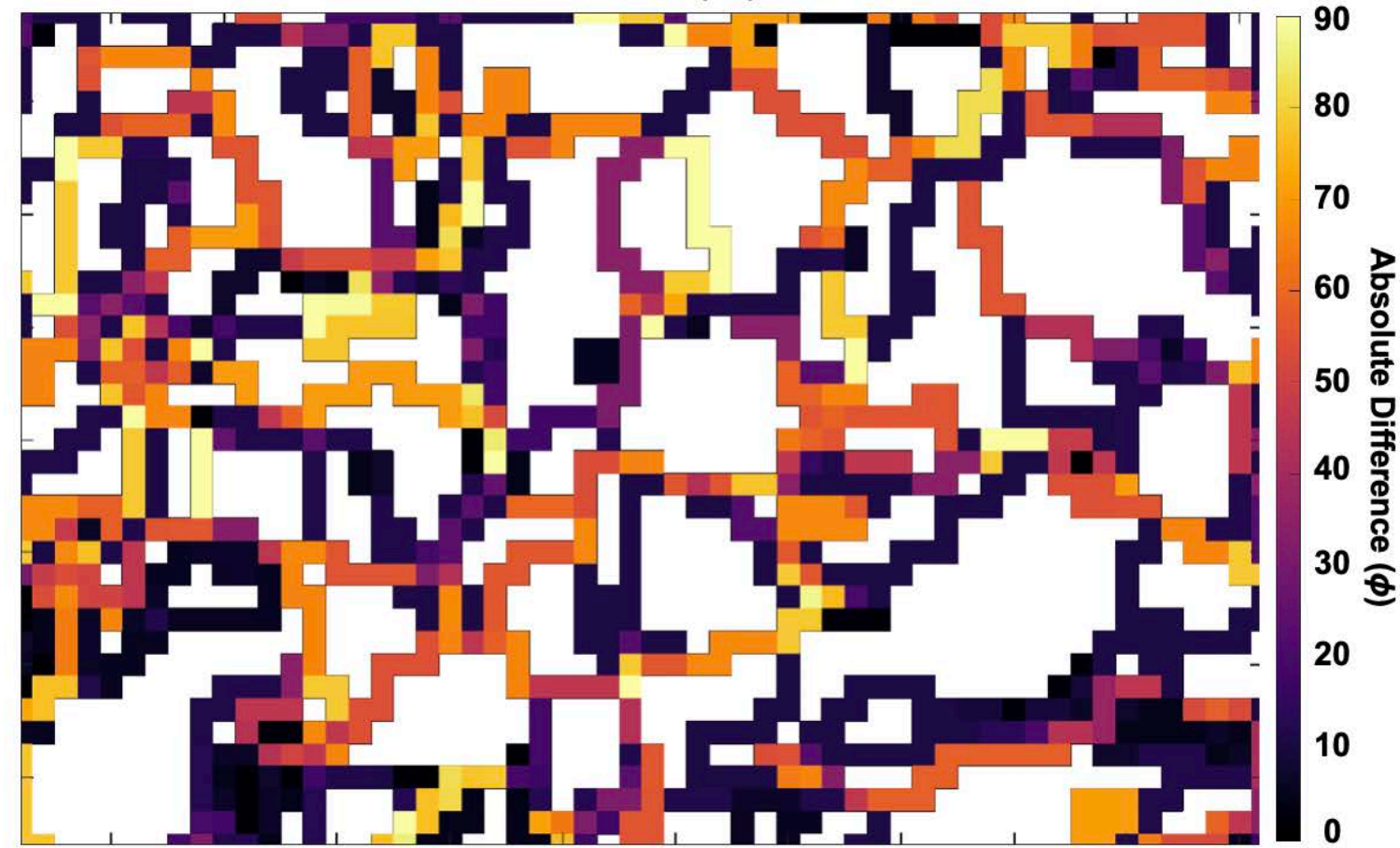

Figure S15. Selection of mapping spatial accuracy for the COF-5 film HR-TEM image shown Figure 2A. (A) Domain map using a $10 \mathrm{~nm}$ accuracy. (B) Grain boundary map using a $10 \mathrm{~nm}$ accuracy. 


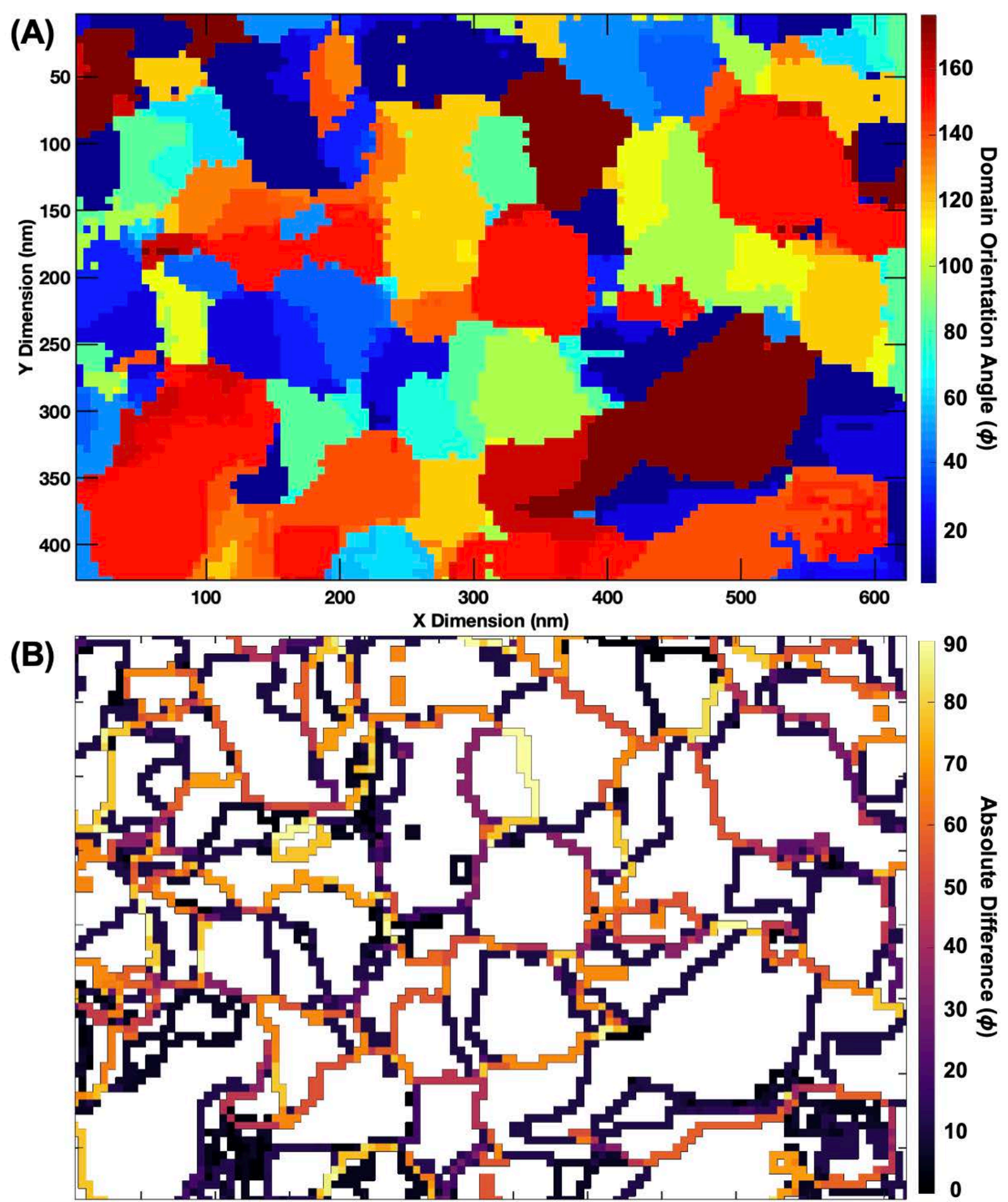

Figure S16. Selection of mapping spatial accuracy for the COF-5 film HR-TEM image shown Figure 2A. (A) Domain map using a $5 \mathrm{~nm}$ accuracy. (B) Grain boundary map using a $5 \mathrm{~nm}$ accuracy. 


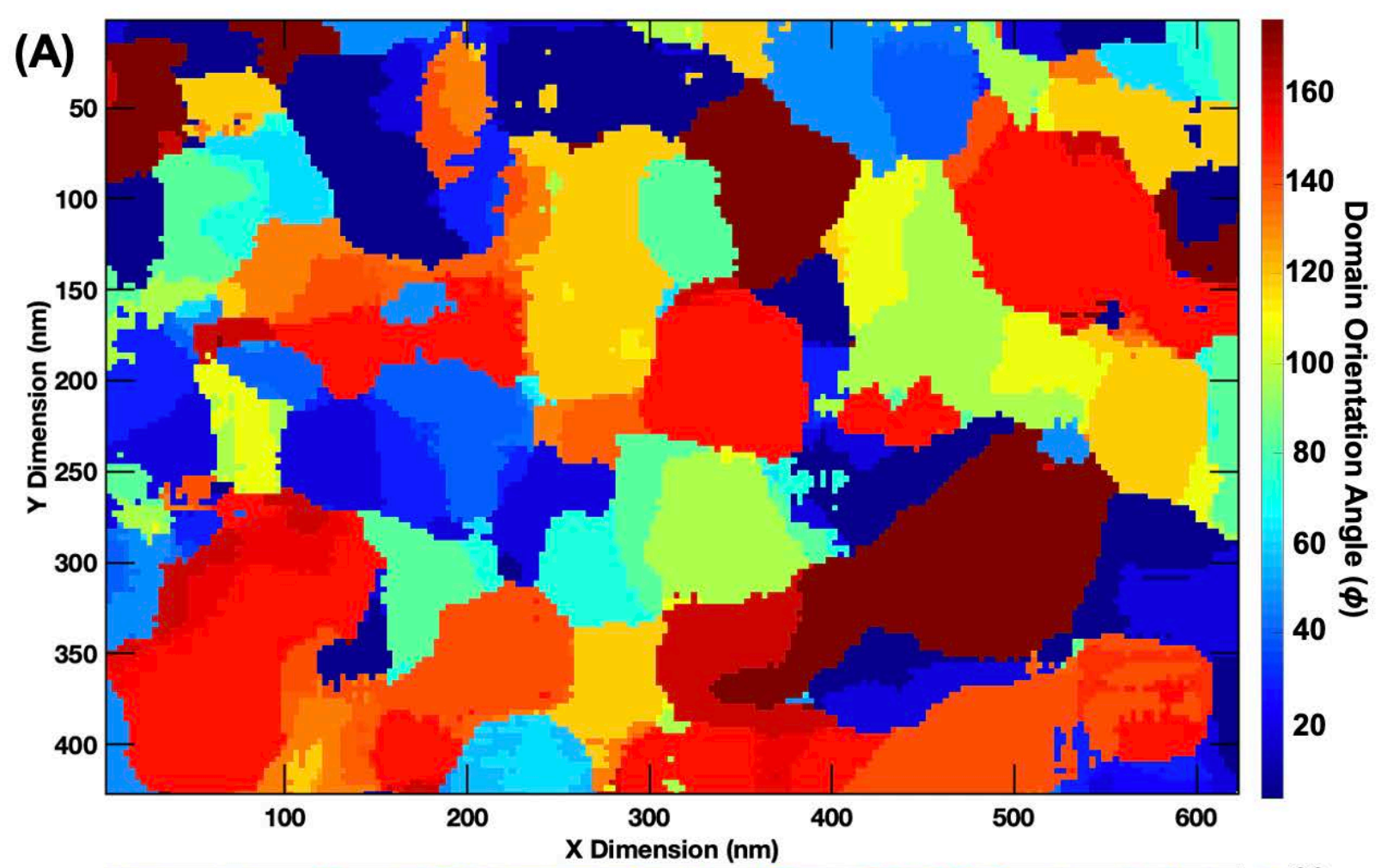

(B)

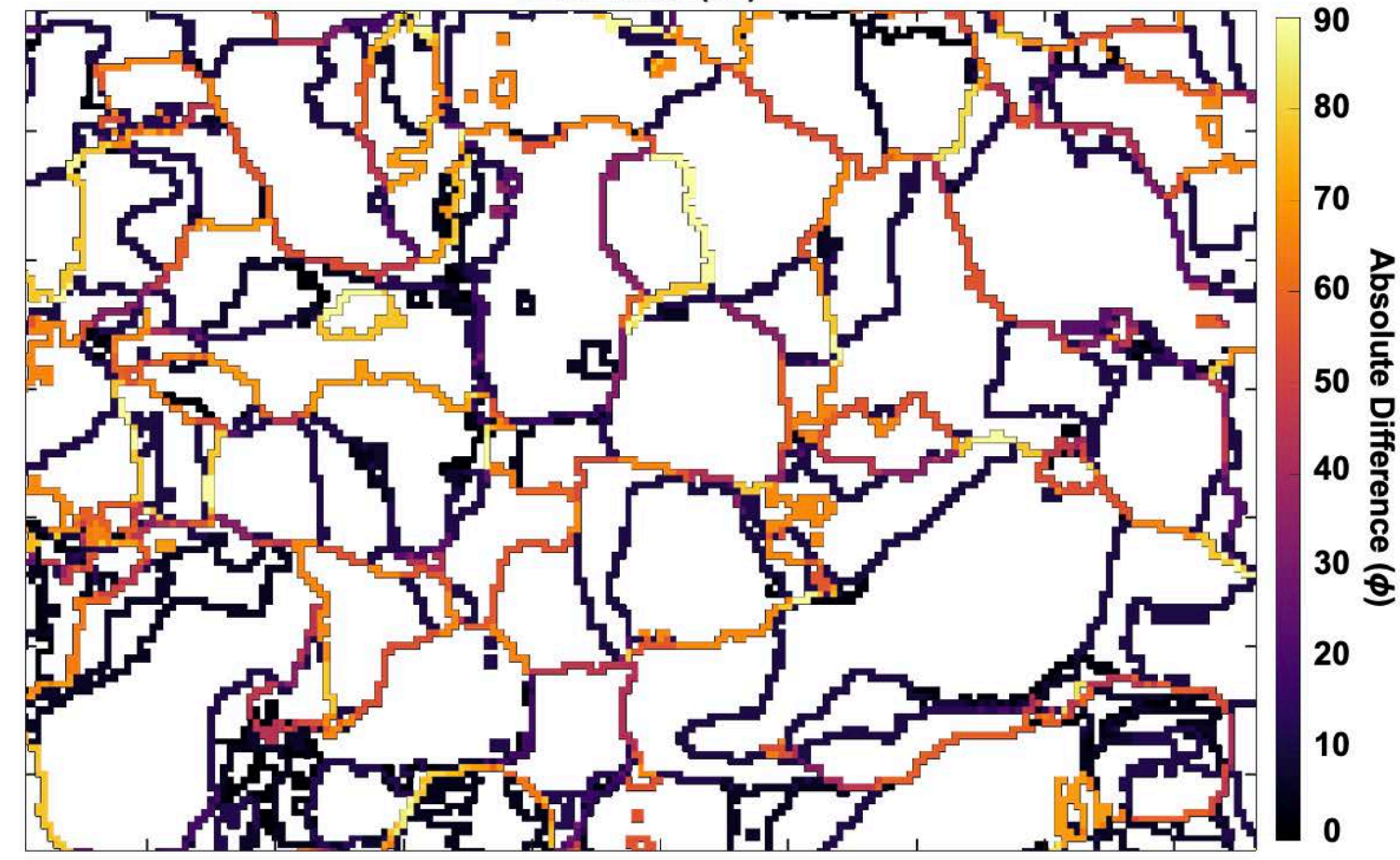

Figure S17. Selection of mapping spatial accuracy for the COF-5 film HR-TEM image shown Figure 2A. (A) Domain map using a $3 \mathrm{~nm}$ accuracy. (B) Grain boundary map using a $3 \mathrm{~nm}$ accuracy. 
VII. MATLAB Script: Selecting Phi Degree Binning
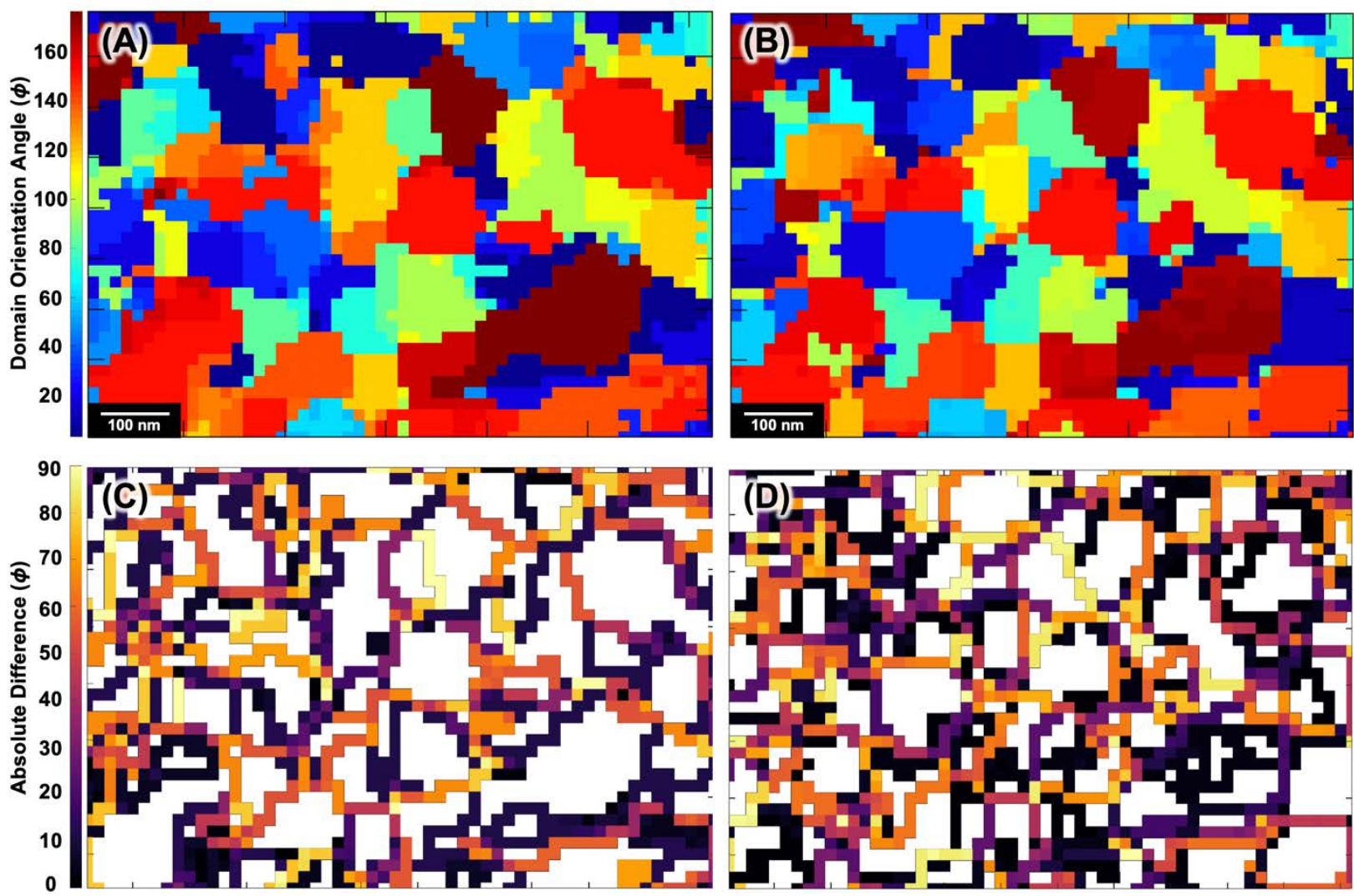

Figure S18. Selection of phi degree binning for the COF-5 film HR-TEM image shown in Figure 2A. (A) Domain orientation map using $10 \mathrm{~nm}$ accuracy, 500 pixel size, and $5^{\circ}$ phi binning. (B) Domain orientation map using $10 \mathrm{~nm}$ accuracy, 500 pixel size, and $3^{\circ}$ phi binning. (C) Corresponding grain boundary map using same parameters as (A). (D) Corresponding grain boundary map using same parameters as (B). 
VIII. MATLAB Script: Domain Mapping

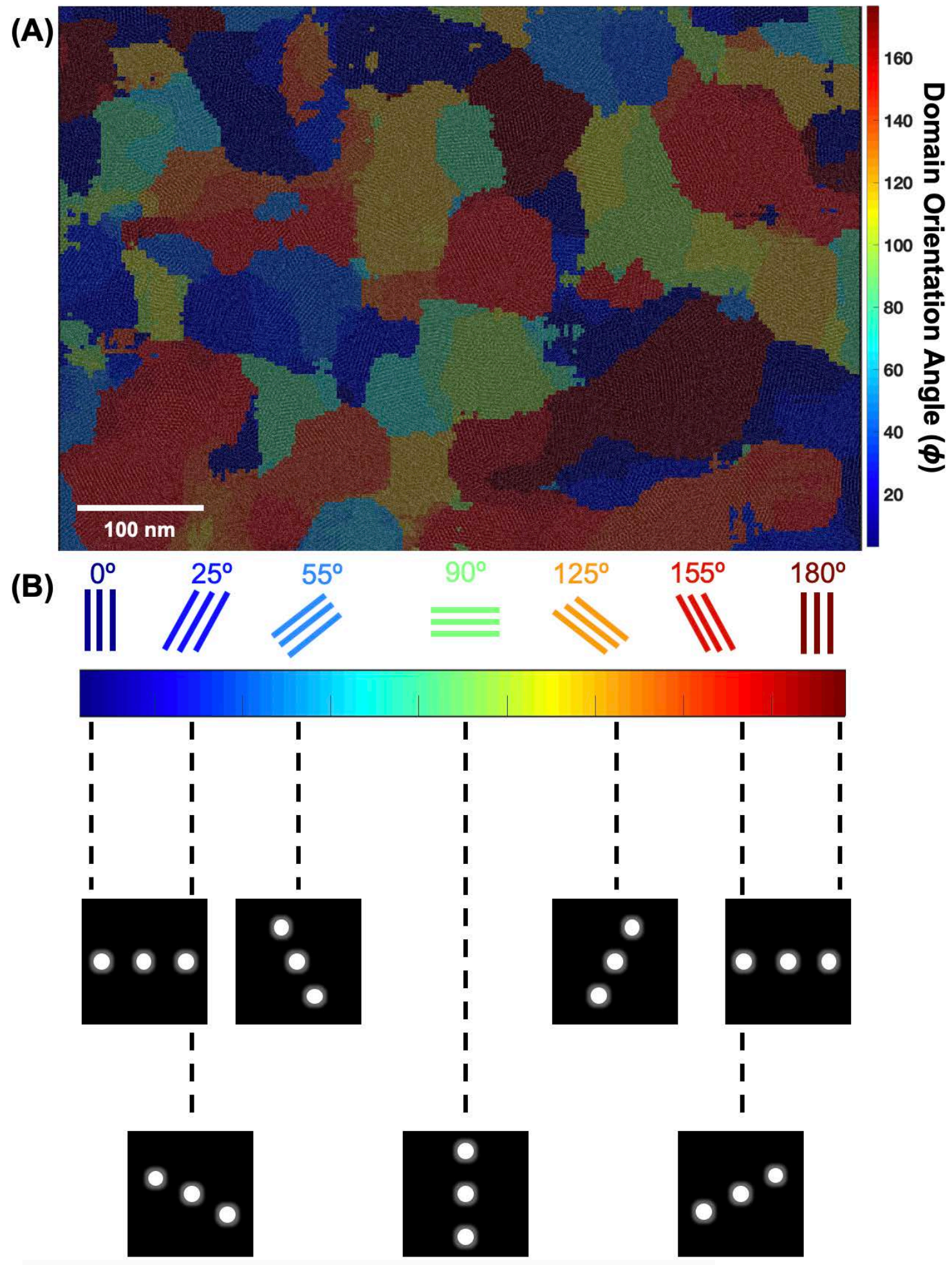

Figure S19. (A) Domain mapped COF-5 film TEM image. (B) Schematic showing the lattice angle and FFT that correspond to the different colors in the mapped image. 


\section{Analysis of COF-5 Grain Boundaries}

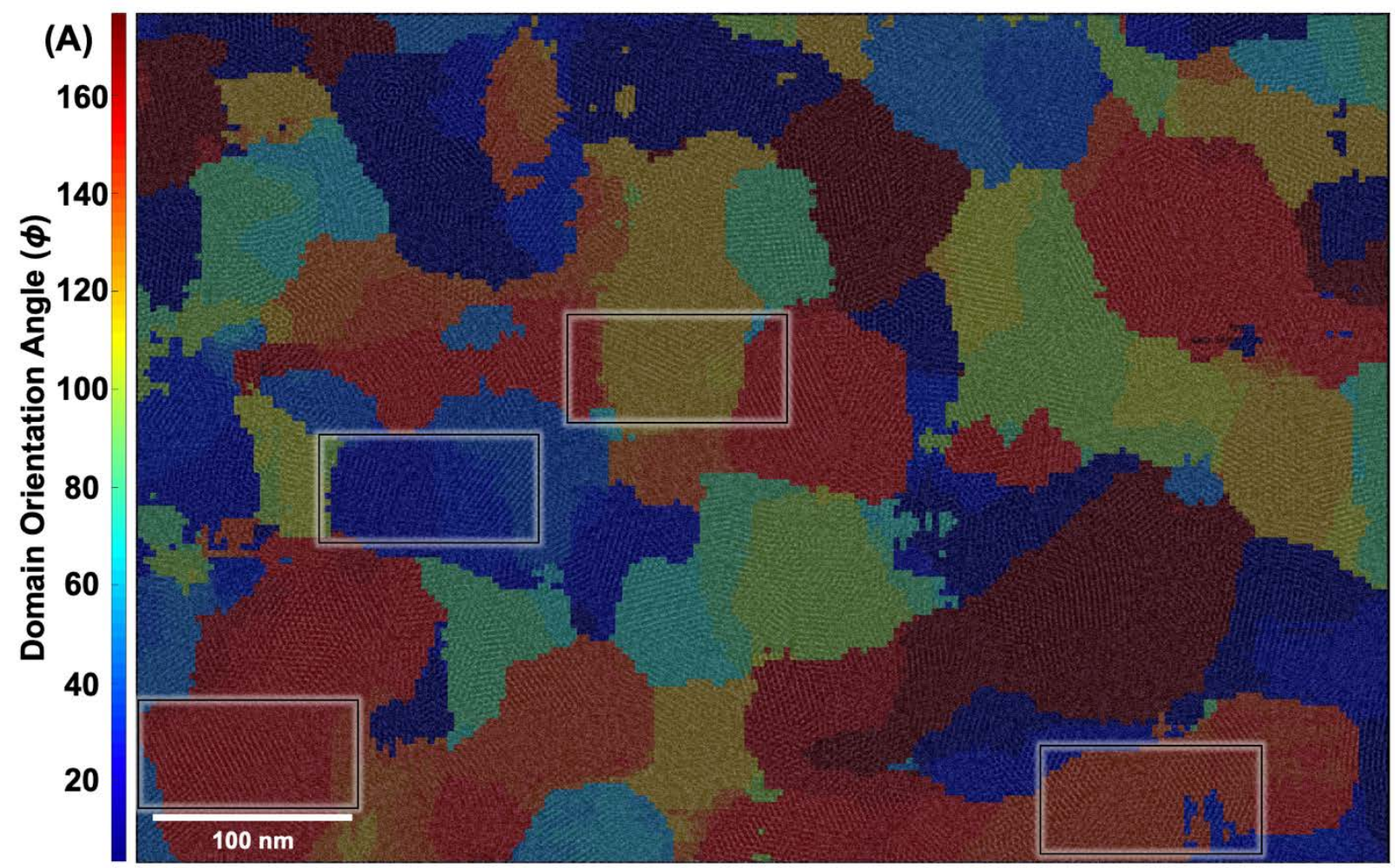

(B)
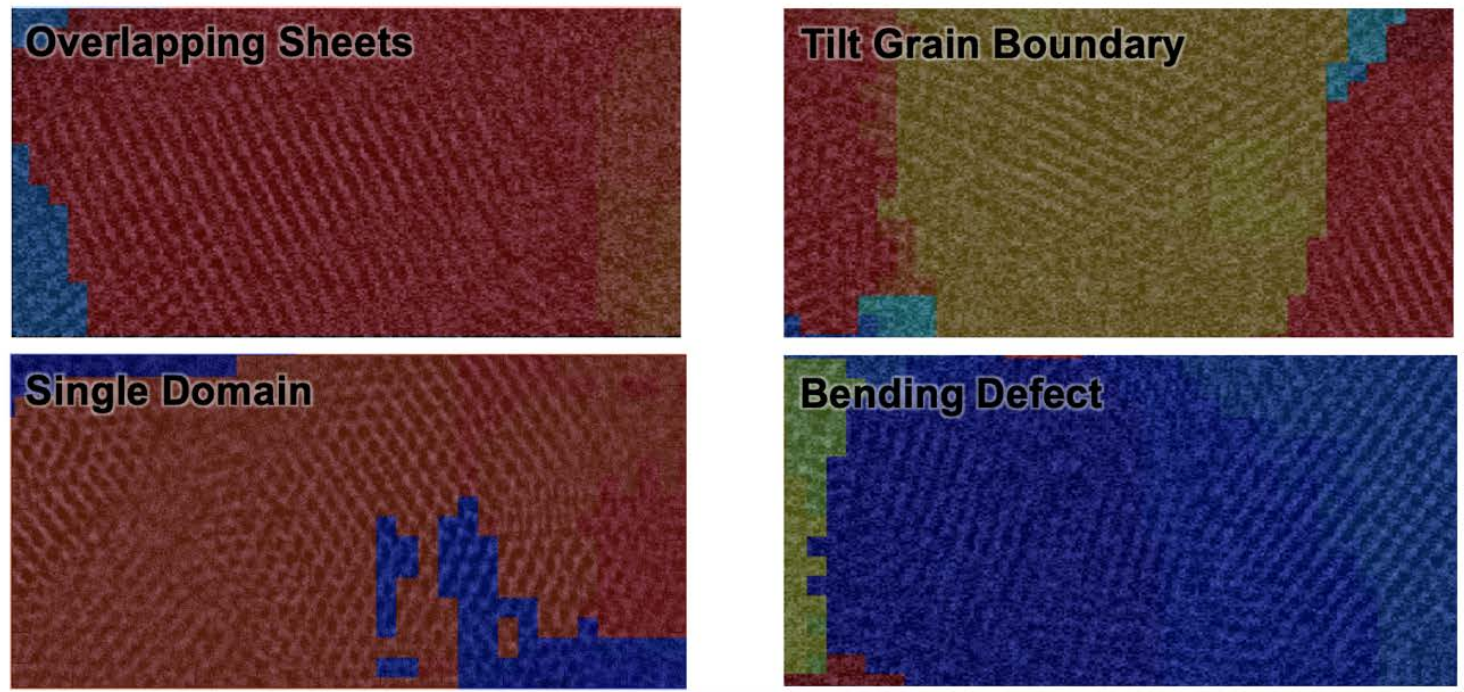

Figure S20. (A) Domain mapped COF-5 film TEM image with boxed regions corresponding to different types of grain boundaries or domains. (B) Magnified images of boxed regions in (A) showing the different types of regions. 


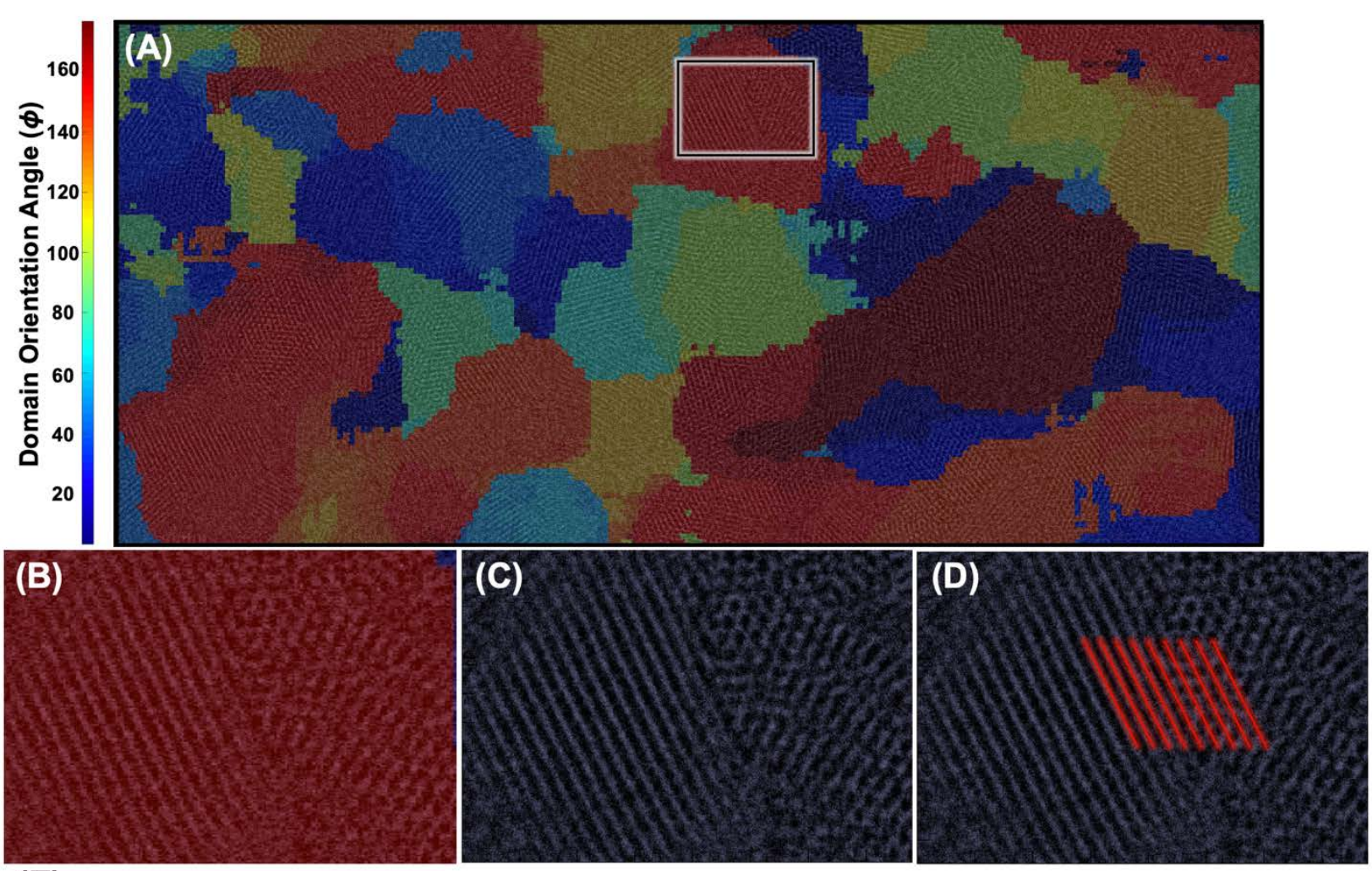

(E)

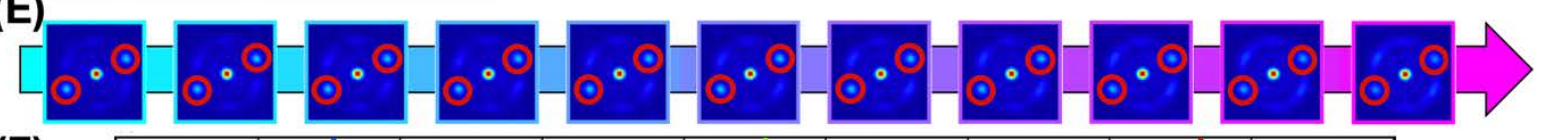

(F)

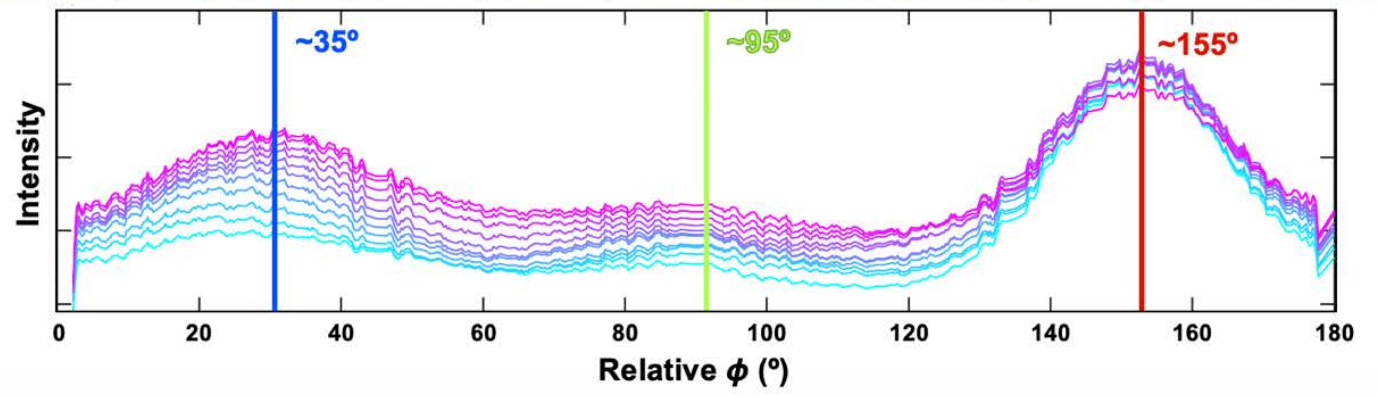

Figure S21. Analysis of Domain Bending. (A) Domain mapped HR-TEM image of COF-5 film shown in Figure 4A. Boxed region displays single-colored domain observed in the image. (B) Magnified image of boxed region in (A) with colored domain map overlaid. (C) Original HR-TEM image of boxed region in (A). (D) Image in (C) with red lines overlaid which correspond to a domain orientation of $155^{\circ}$. (E) FFT patterns of regions moving from left to right (cyan to magenta) in magnified image. (F) Plot displaying the relative phi intensities moving from left to right (cyan to magenta) in the magnified image which shows that the entire region has spots of the highest intensity at the same phi location. As we move further right in the region, spots at $35^{\circ}$ and $95^{\circ}$ appear that are of lower intensity. These spots are exactly $60^{\circ}$ apart from the highest intensity spots at $155^{\circ}$ and are therefore indicative of a change from two-fold to six-fold symmetry which is shown in the TEM image with the change in lattice from lines to hexagons as we move from left to right. 


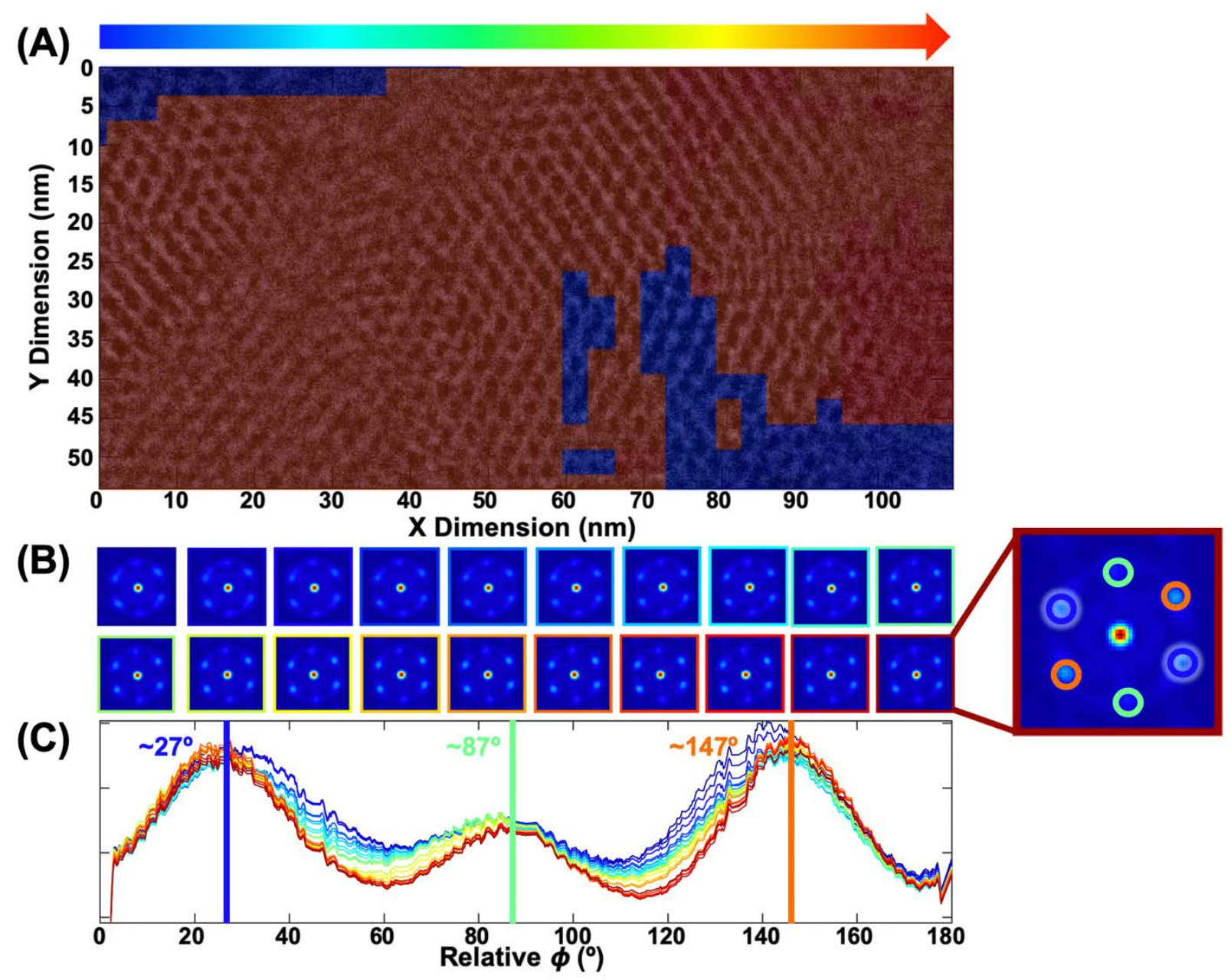

Figure S22. (A) Zoomed in region of the COF-5 film HR-TEM image showing predominately a single crystalline domain. (B) FFT patterns of regions moving from left to right (blue to red) in the cropped image shown in (A). (C) Plot displaying the relative phi intensities which show the changes in grain orientation throughout the image. 


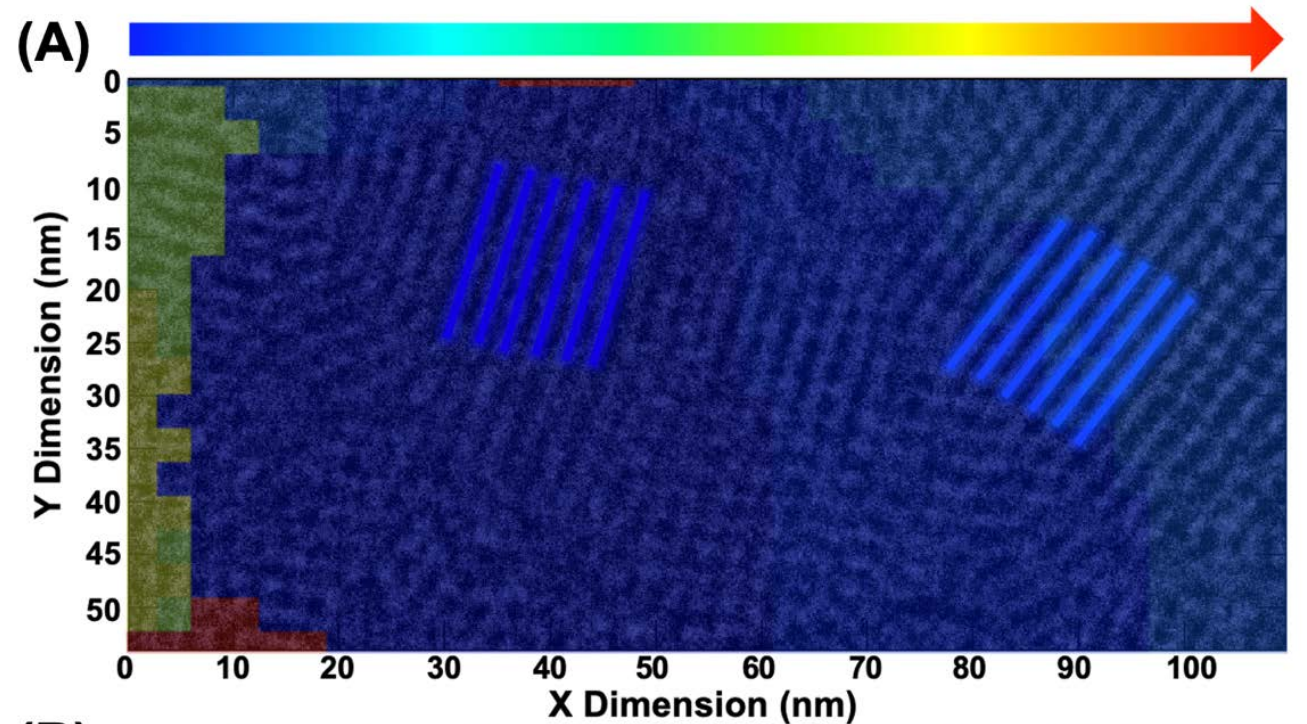

(B)

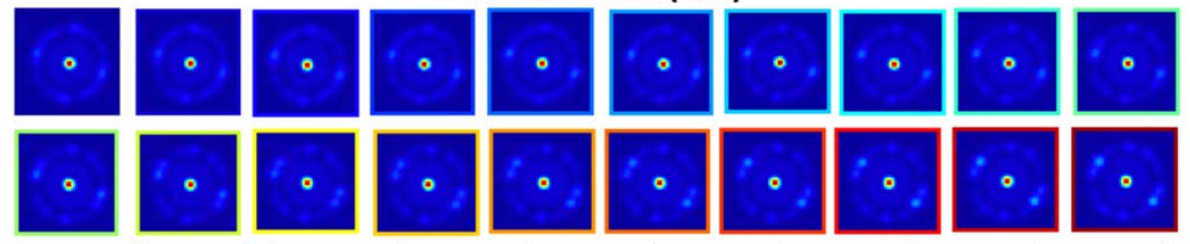

(C)

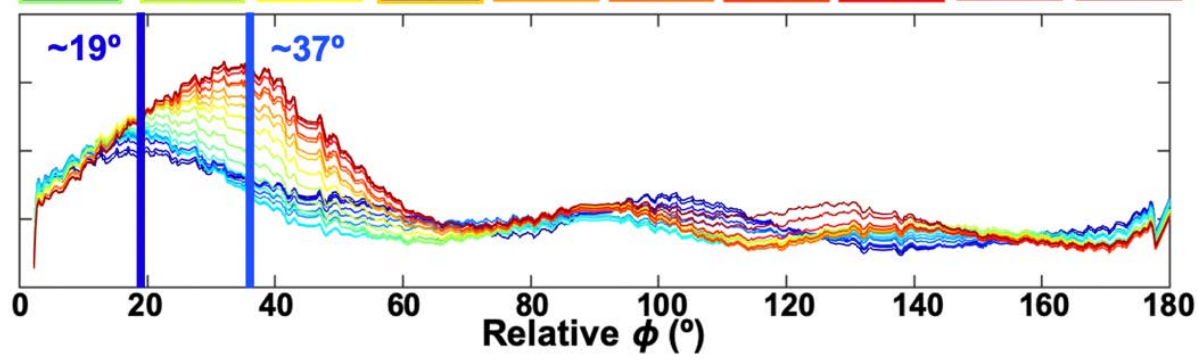

Figure S23. (A) Zoomed in region of the COF-5 film HR-TEM image showing a bending defect. (B) FFT patterns of regions moving from left to right (blue to red) in the cropped image shown in (A). (C) Plot displaying the relative phi intensities which show the changes in grain orientation throughout the image. 


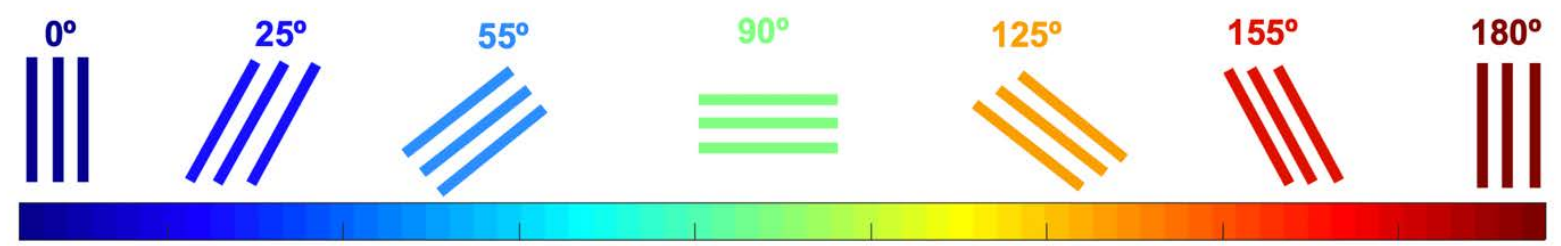

Domain Orientation Angle ( $\phi)$

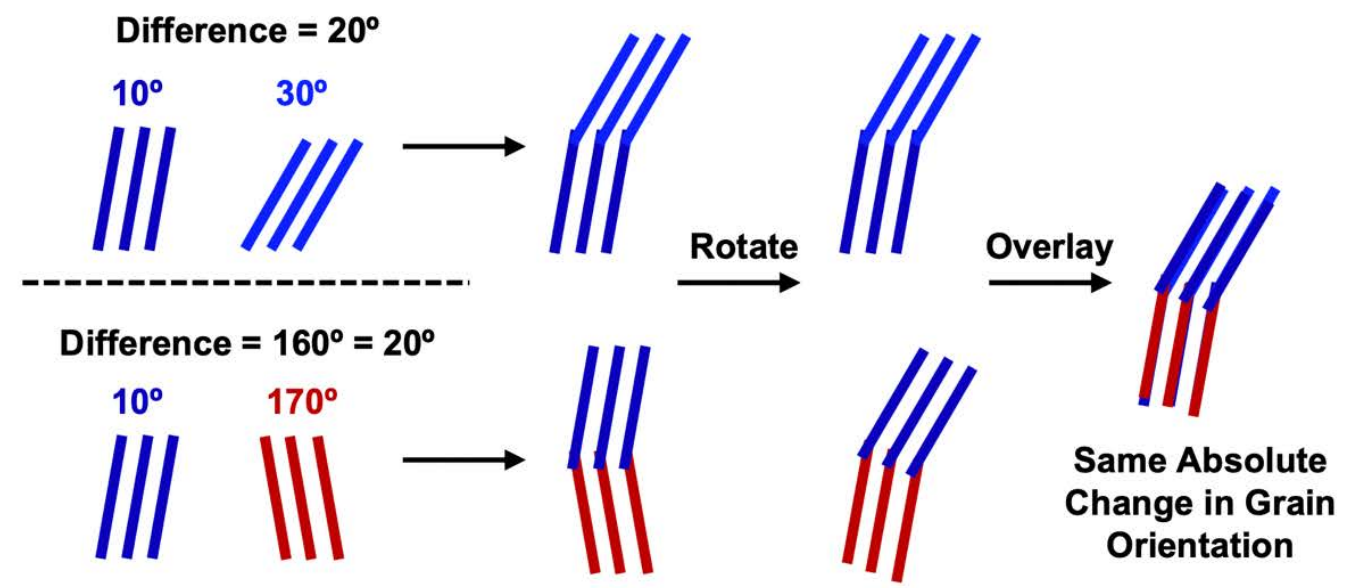

Figure S24. Schematic showing the absolute difference in grain orientation between adjacent crystalline domains. A domain with an orientation of $10^{\circ}$ that is adjacent to a $30^{\circ}$ domain has the same absolute change in grain orientation as a domain with an orientation of $10^{\circ}$ that is adjacent to a $170^{\circ}$ domain because in both cases the absolute difference is $20^{\circ}$. 


\section{Mapping Other Boronate Ester-Linked COFs}

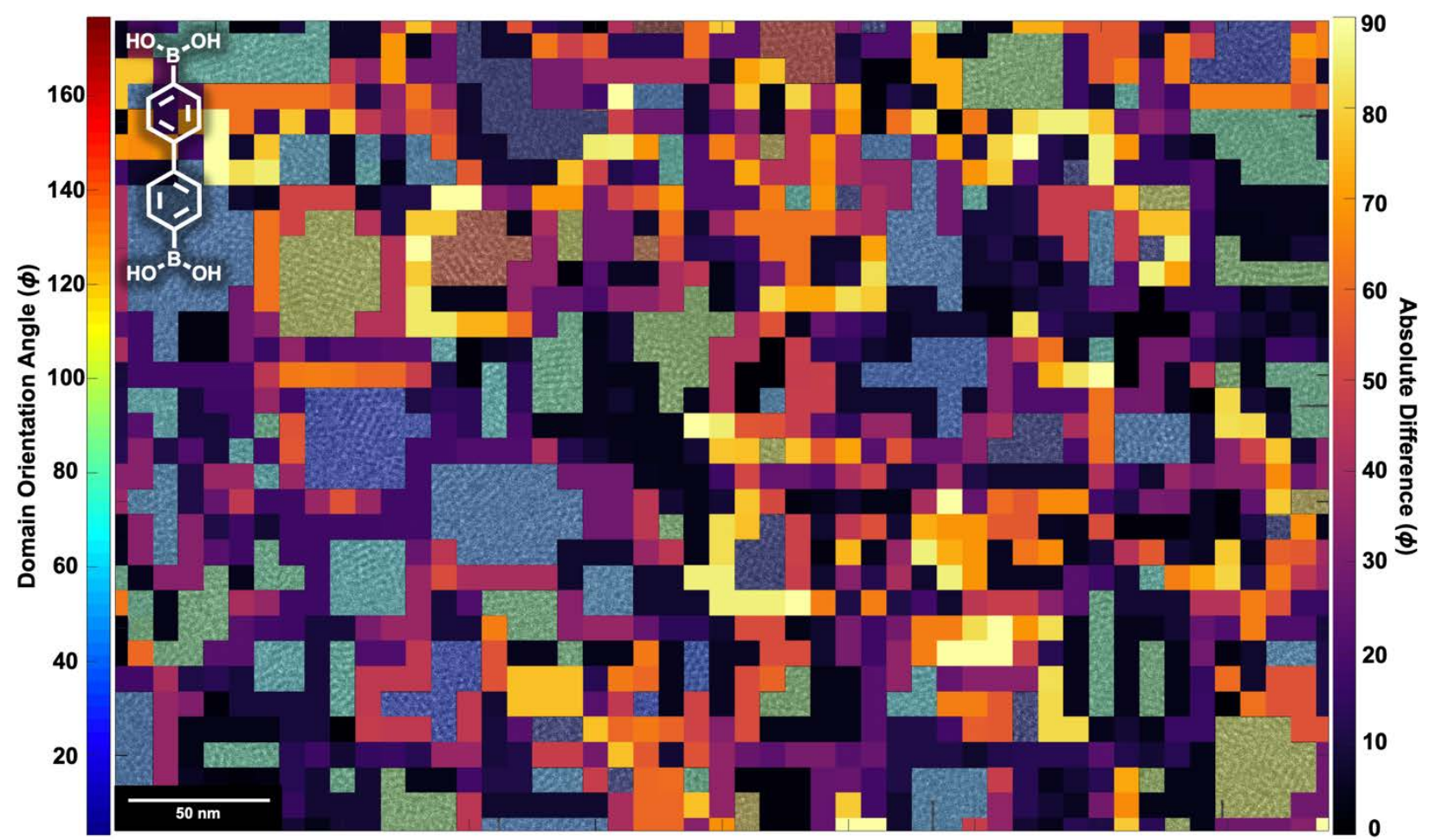

Figure S25. HR-TEM image of a COF-10 film grown on a graphene coated TEM (Figure 1C) with domain orientation map and grain boundary map overlaid. Dose Rate: $17.35 \mathrm{e}^{-} \AA^{-2} \mathrm{~s}^{-1}$ Cumulative Dose: $17.28 \mathrm{e}^{-} \AA^{-2}$. Mapping parameters: $8 \mathrm{~nm}$ accuracy, 500 pixel frame, $5^{\circ} \mathrm{phi}$ binning. 


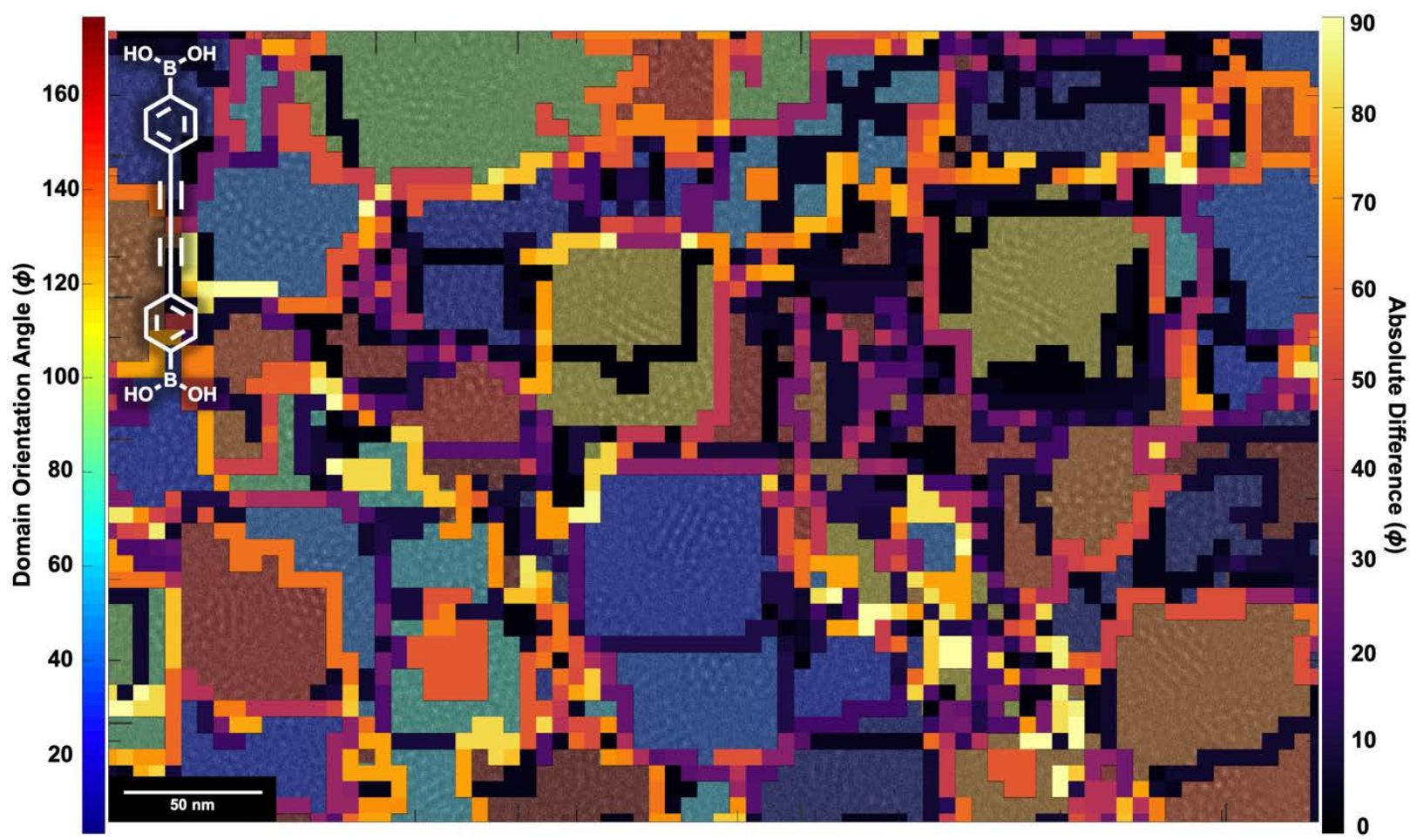

Figure S26. HR-TEM image of a DPB-COF film grown on a graphene coated TEM (Figure 1D) with domain orientation map and grain boundary map overlaid. Dose Rate: $16.45 \mathrm{e}^{-} \AA^{-2} \mathrm{~s}^{-1}$ Cumulative Dose: $6.40 \mathrm{e}^{-} \AA^{-2}$. Mapping parameters: $5 \mathrm{~nm}$ accuracy, 750 pixel frame, $5^{\circ}$ phi binning. 


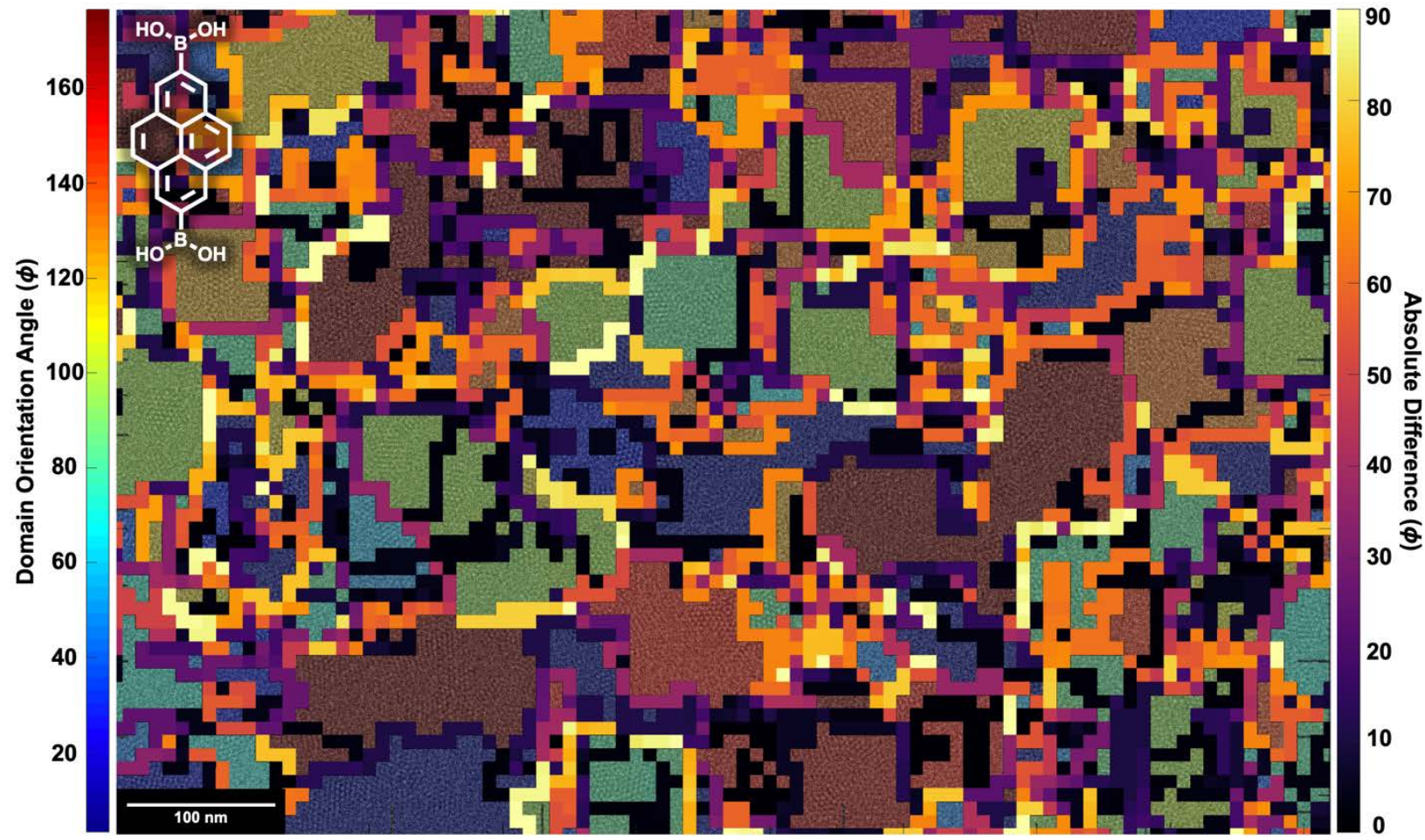

Figure S27. HR-TEM image of a TP-COF film grown on a graphene coated TEM (Figure 1D) with domain orientation map and grain boundary map overlaid. Dose Rate: $2.12 \mathrm{e}^{-} \AA^{-2} \mathrm{~s}^{-1}$ Cumulative Dose: $0.41 \mathrm{e}^{-} \AA^{-2}$. Mapping parameters: $8 \mathrm{~nm}$ accuracy, 500 pixel frame, $5^{\circ}$ phi binning. 


\section{Mapping Other Materials}

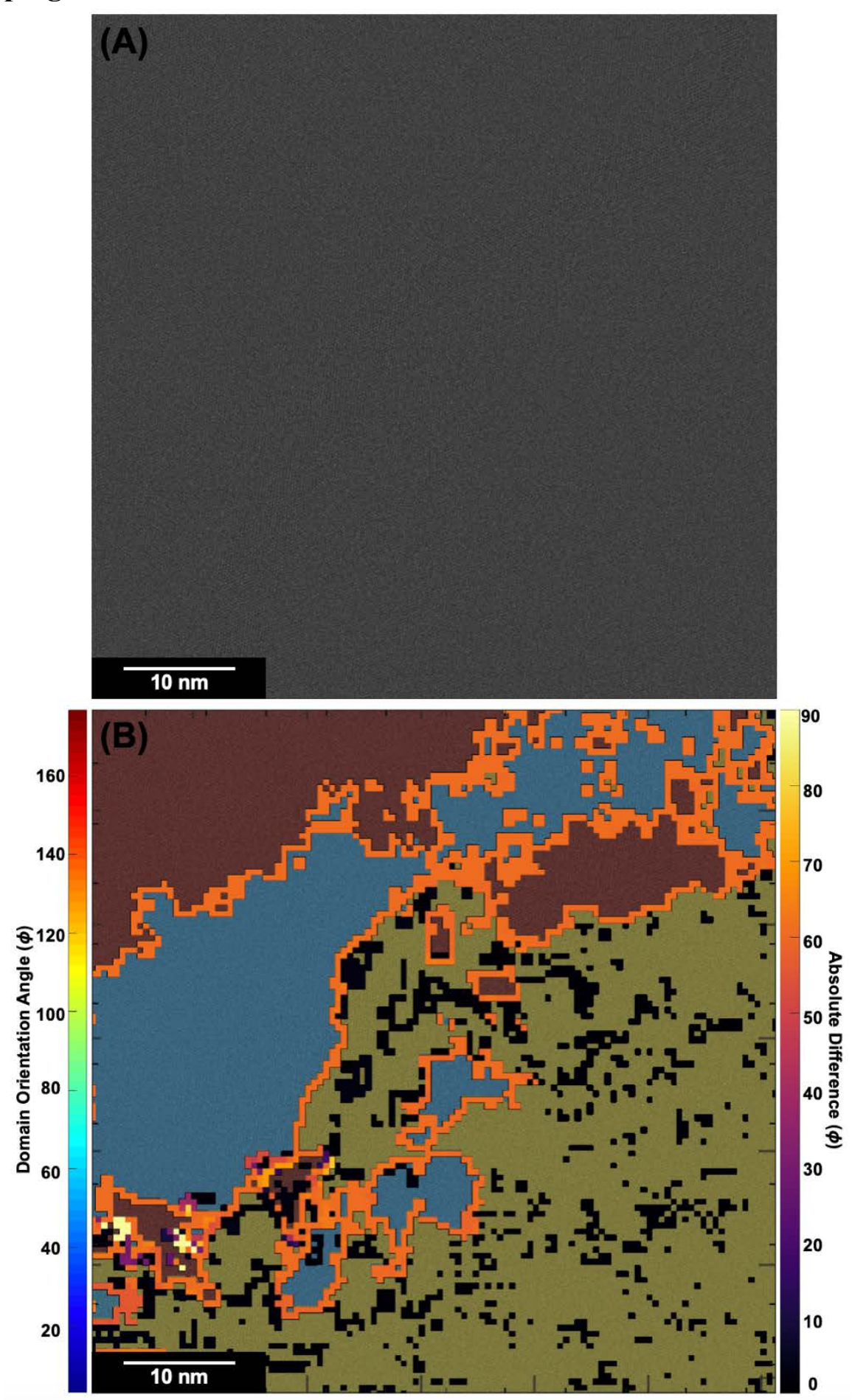

Figure S28. (A) HR-TEM image of a $\mathrm{MoS}_{2}$ region collected using dose fractionation within 36 frames with a total acquisition time of $0.45 \mathrm{~s}$ and a dose rate of $17 \mathrm{e}^{-} \mathrm{px}^{-1} \mathrm{~s}^{-1}{ }^{6}$ (B) Domain orientation map and grain boundary map overlaid on the HR-TEM image. Mapping parameters: $0.5 \mathrm{~nm}$ accuracy, 250 pixel frame, $5^{\circ}$ phi binning. 


\section{Grazing-Incidence Wide-Angle X-Ray Scattering}
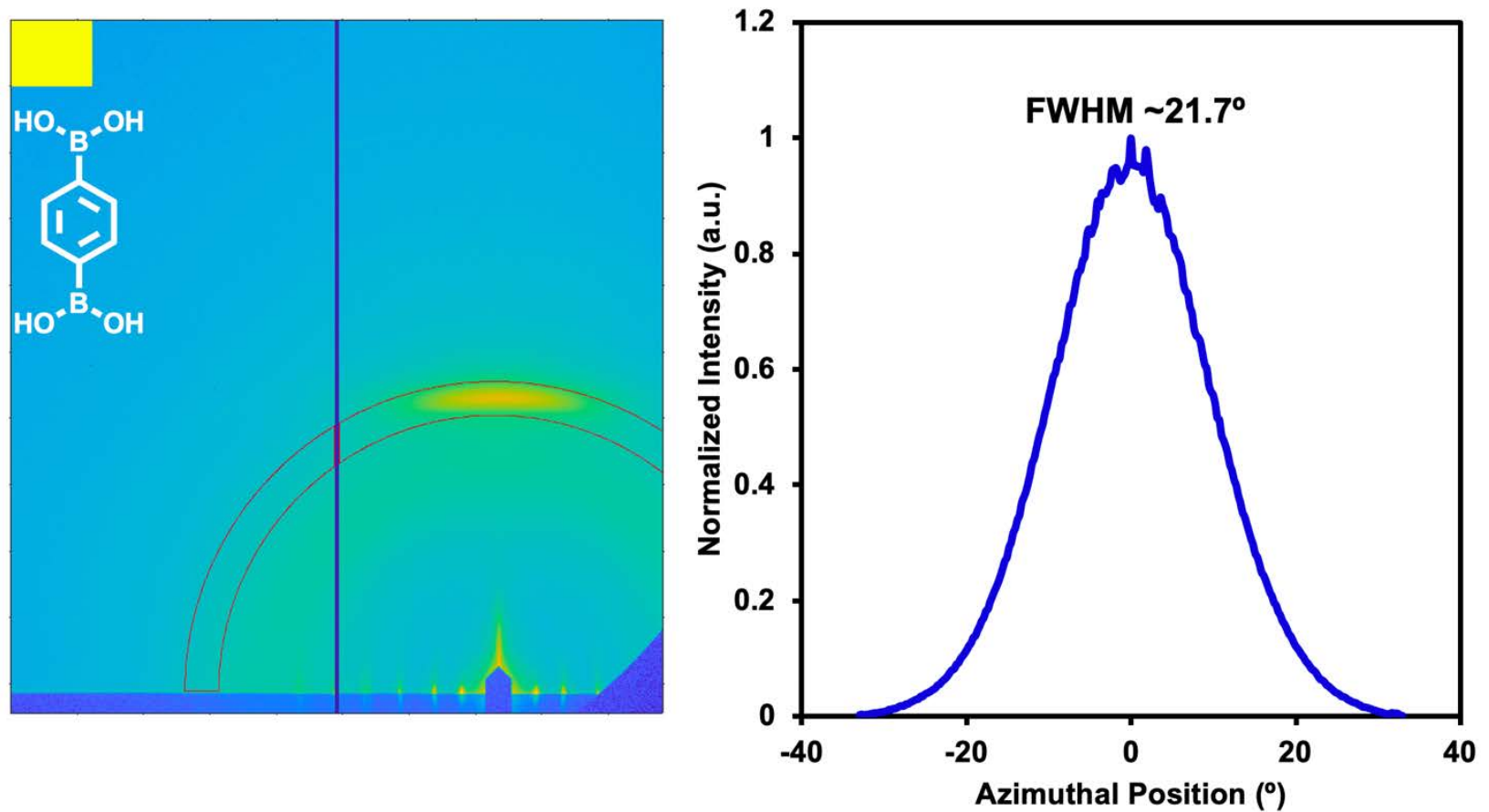

Figure S29. Estimate of the angular distribution using the azimuthal position of the (001) peak in the GIWAXS pattern. GIWAXS pattern for COF-5 with a mask (marked in red) selecting the (001) peak (left). Normalized, background subtracted line cut of the (001) peak with a measured FWHM of $\sim 21.7^{\circ}$ (right). 

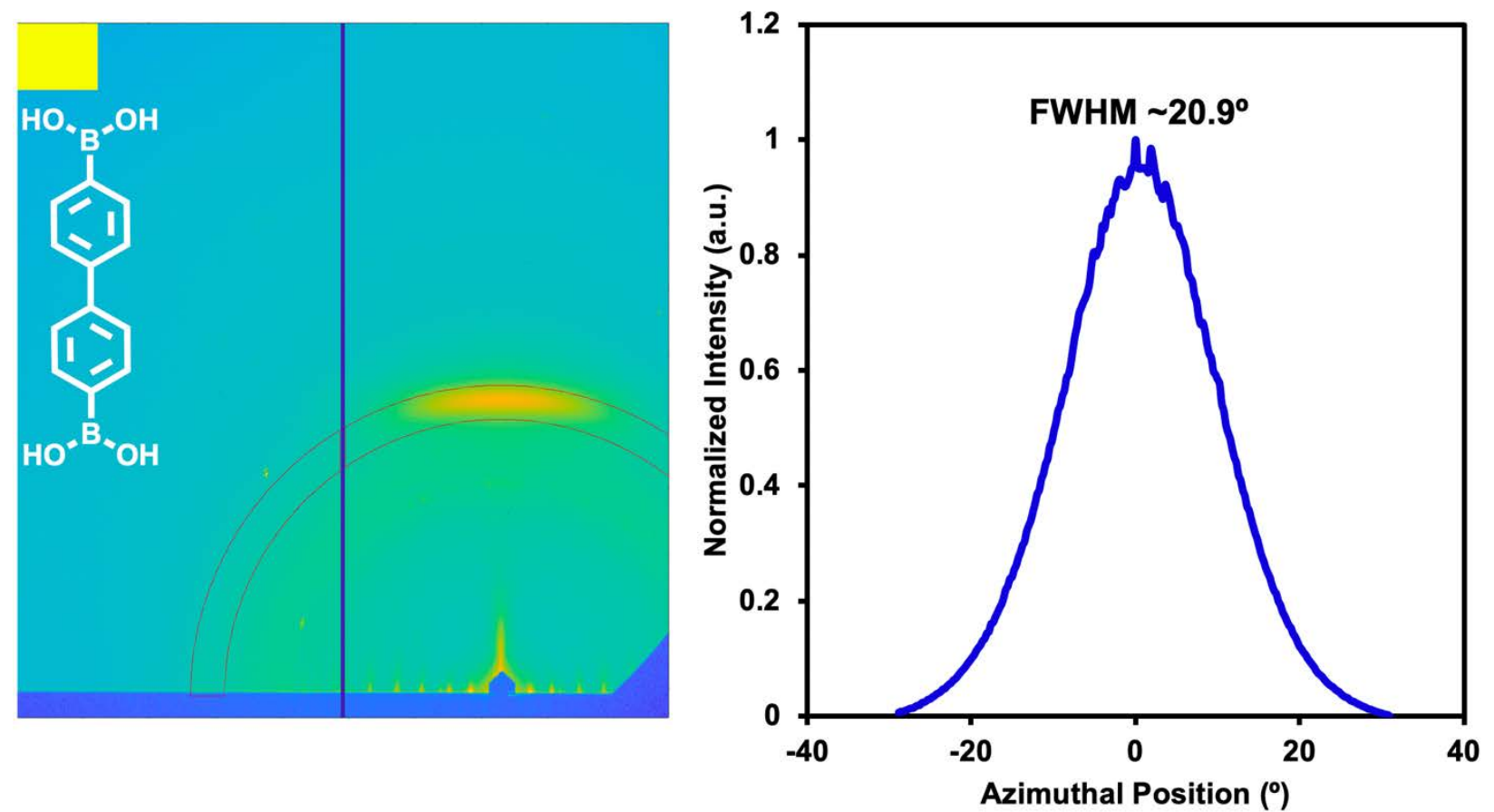

Figure S30. Estimate of the angular distribution using the azimuthal position of the (001) peak in the GIWAXS pattern. GIWAXS pattern for COF-10 with a mask (marked in red) selecting the (001) peak (left). Normalized, background subtracted line cut of the (001) peak with a measured FWHM of $\sim 20.9^{\circ}$ (right). 

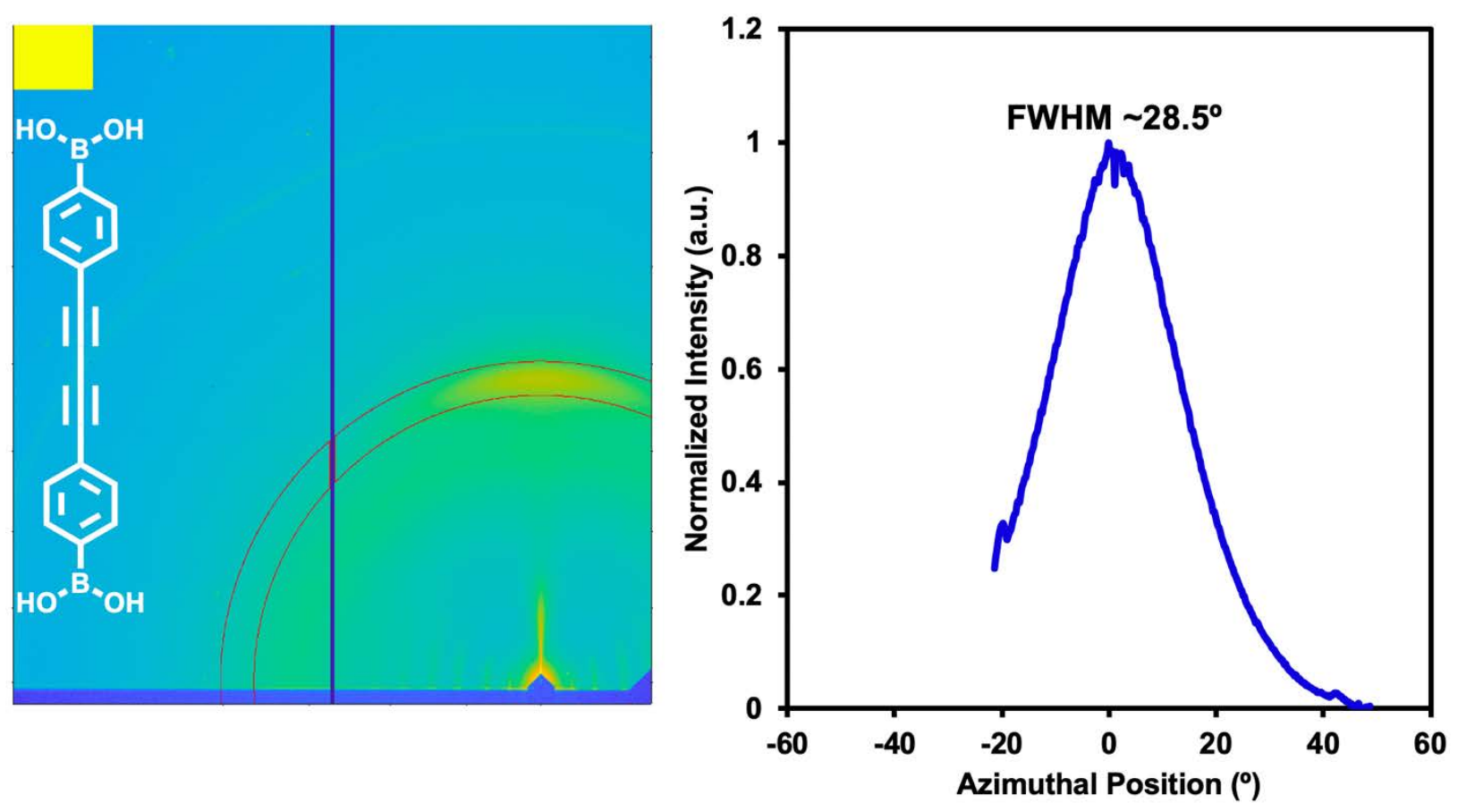

Figure S31. Estimate of the angular distribution using the azimuthal position of the (001) peak in the GIWAXS pattern. GIWAXS pattern for DPB-COF with a mask (marked in red) selecting the (001) peak (left). Normalized, background subtracted line cut of the (001) peak with a measured FWHM of $\sim 28.5^{\circ}$ (right). 

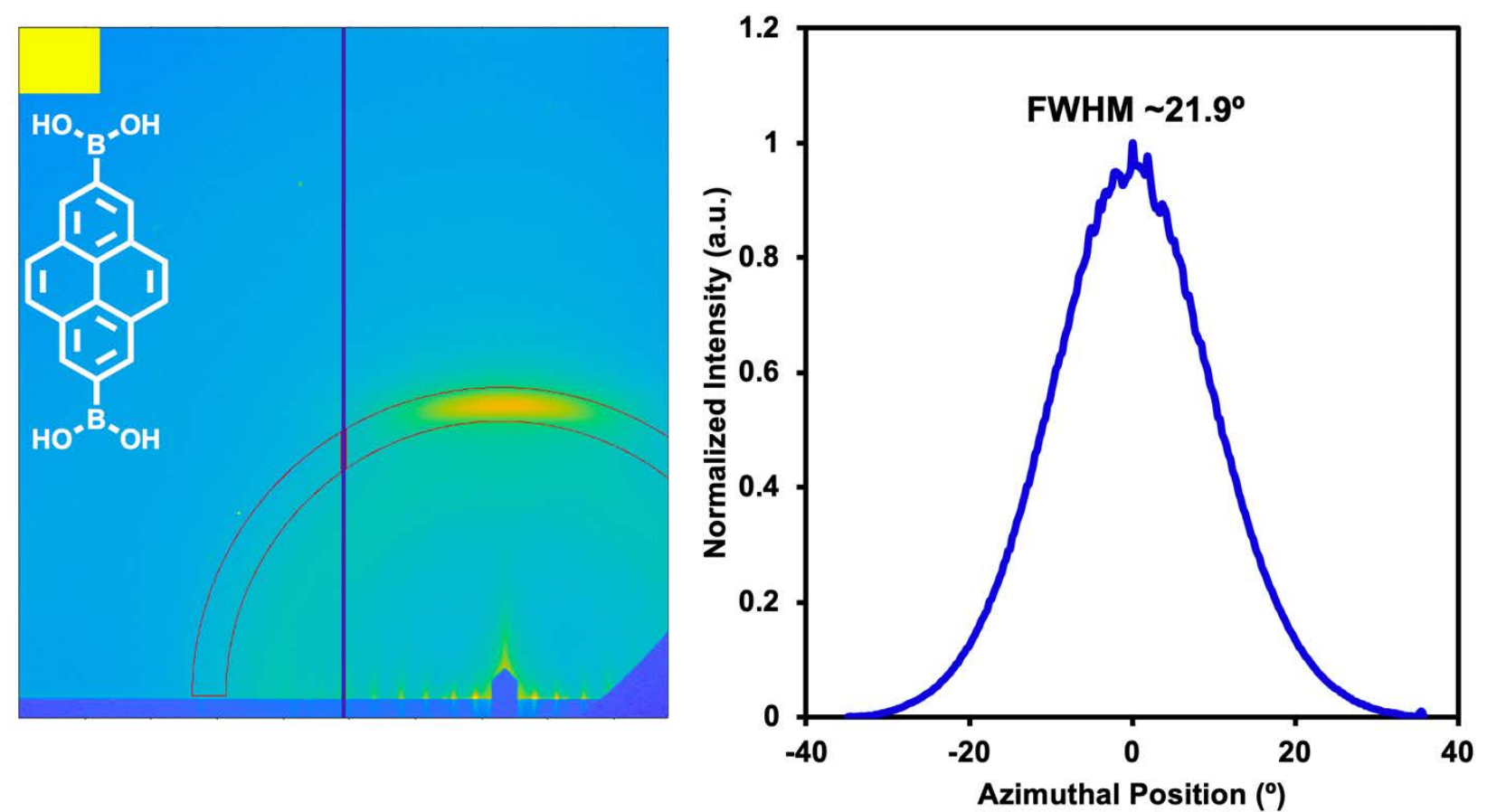

Figure S32. Estimate of the angular distribution using the azimuthal position of the (001) peak in the GIWAXS pattern. GIWAXS pattern for TP-COF with a mask (marked in red) selecting the (001) peak (left). Normalized, background subtracted line cut of the (001) peak with a measured FWHM of $\sim 21.9^{\circ}$ (right). 


\section{Atomic Force Microscopy}
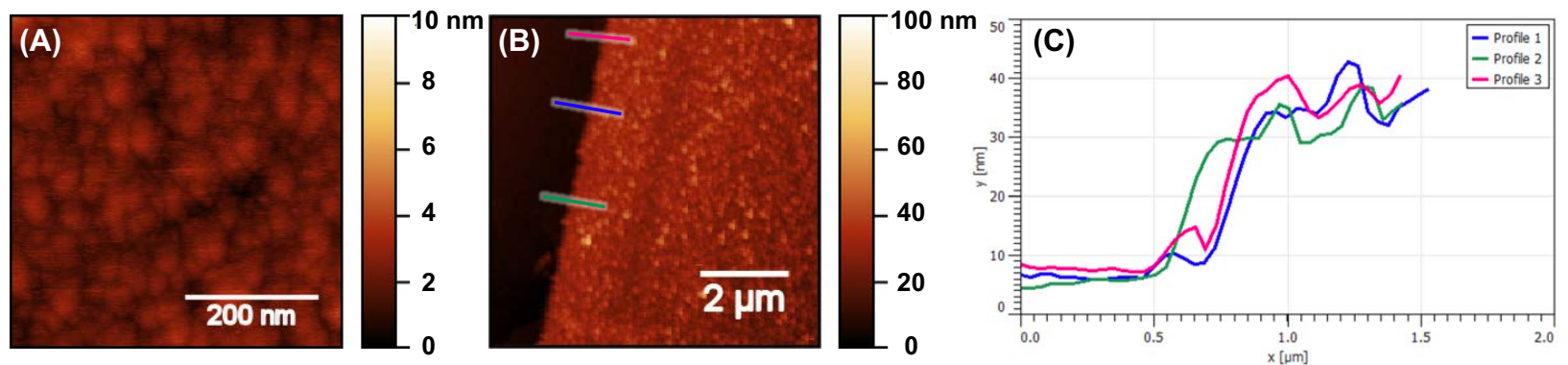

Figure S33. AFM images of COF-5 films. (A) Zoomed in region showing a surface roughness of less than $5 \mathrm{~nm}$. (B) The film was scratched with a razor blade and imaged at the edge of the scratch to determine its thickness. (C) Line profiles across the edge display that the films are approximately $30 \mathrm{~nm}$ thick.
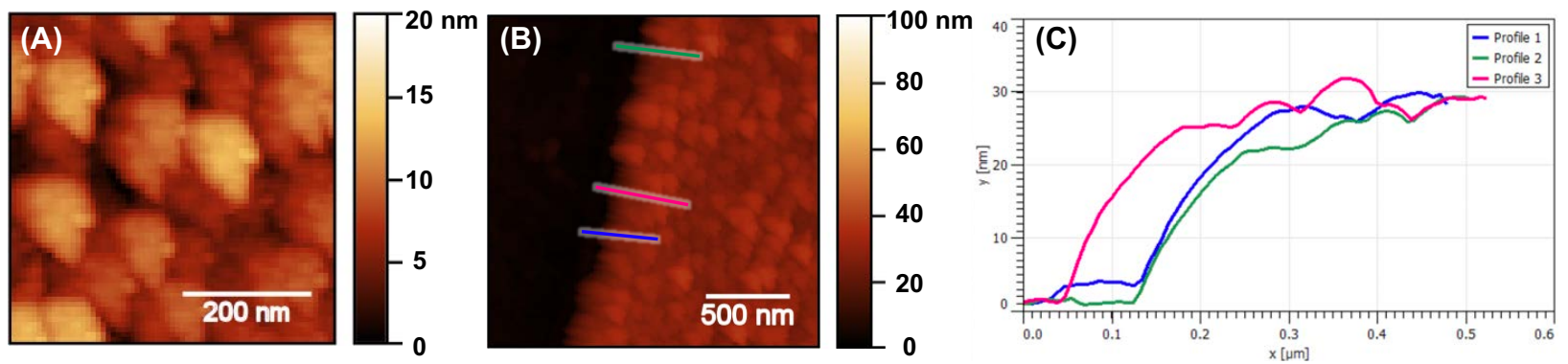

Figure S34. AFM images of COF-10 films. (A) Zoomed in region showing a surface roughness of less than $15 \mathrm{~nm}$. (B) The film was scratched with a razor blade and imaged at the edge of the scratch to determine its thickness. (C) Line profiles across the edge display that the films are approximately $30 \mathrm{~nm}$ thick.
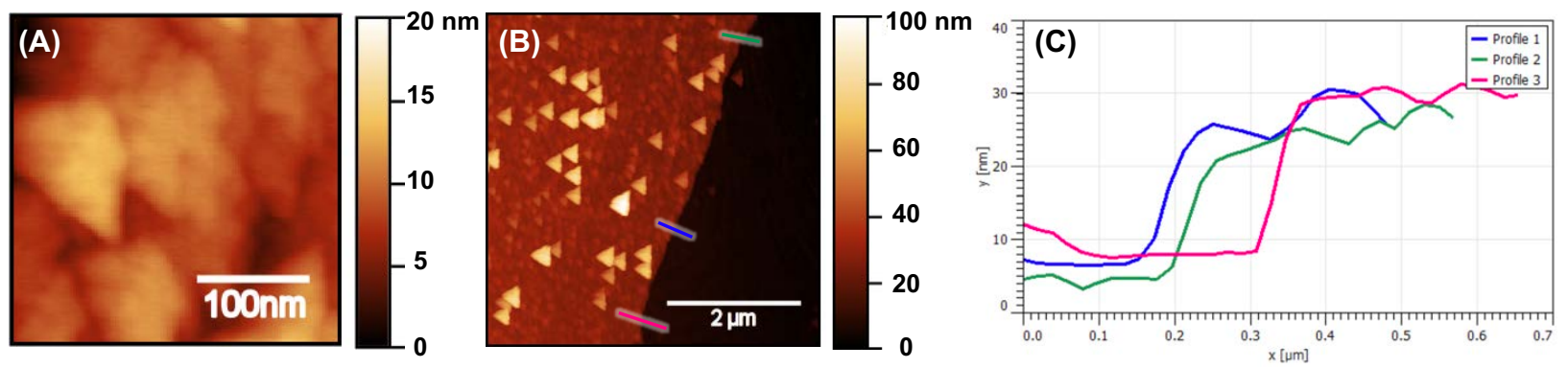

Figure S35. AFM images of TP COF films. (A) Zoomed in region showing a surface roughness of less than $10 \mathrm{~nm}$. (B) The film was scratched with a razor blade and imaged at the edge of the scratch to determine its thickness. (C) Line profiles across the edge display that the films are approximately $20 \mathrm{~nm}$ thick. 

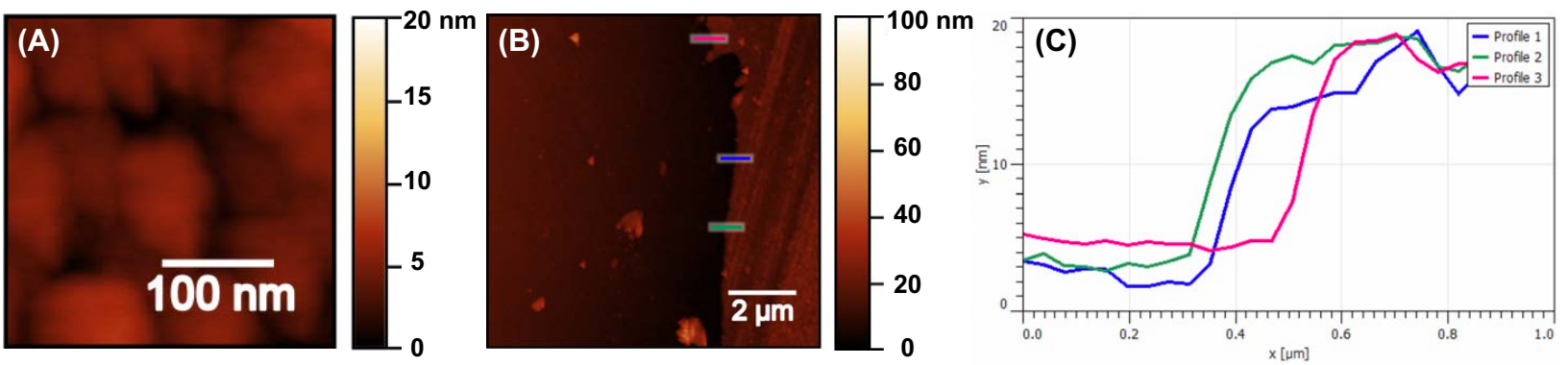

Figure S36. AFM images of DPB COF films. (A) Zoomed in region showing a surface roughness of less than $10 \mathrm{~nm}$. (B) The film was scratched with a razor blade and imaged at the edge of the scratch to determine its thickness. (C) Line profiles across the edge display that the films are approximately $15 \mathrm{~nm}$ thick.

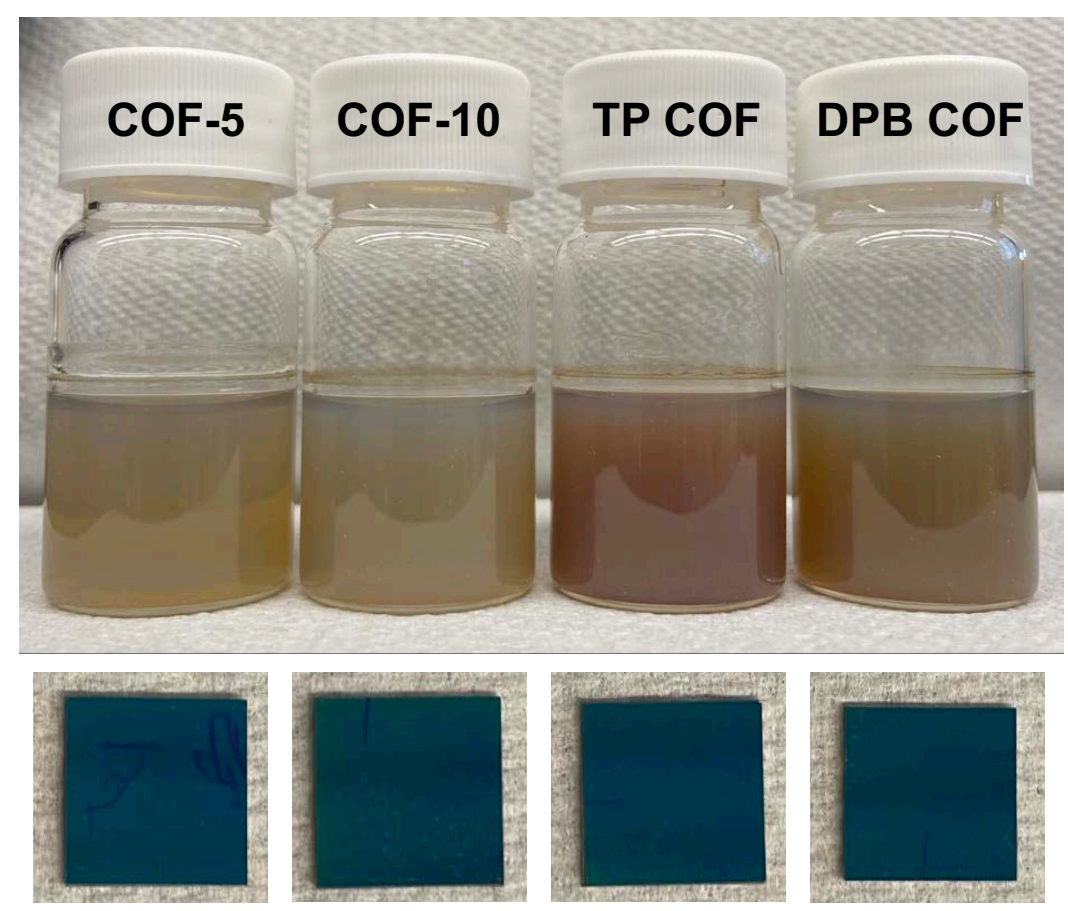

Figure S37. COF colloid solutions (top) and corresponding films grown on $\mathrm{SiO}_{2}$ substrates (bottom). Scratches on the $\mathrm{SiO}_{2}$ substrates were made with a razor blade after film growth for film thickness analysis. 


\section{References}

1. Wan, S.; Guo, J.; Kim, J.; Ihee, H.; Jiang, D. A Belt-Shaped, Blue Luminescent, and Semiconducting Covalent Organic Framework. Angew. Chem. Int. Ed. 2008, 47 (46), 8826-8830.

2. Spitler, E. L.; Koo, B. T.; Novotney, J. L.; Colson, J. W.; Uribe-Romo, F. J.; Gutierrez, G. D.; Clancy, P.; Dichtel, W. R. A 2D Covalent Organic Framework with 4.7-nm Pores and Insight into Its Interlayer Stacking. J. Am. Chem. Soc. 2011, 133 (48), 19416-19421.

3. Accelrys, 4.4 ed., Accelrys Software, San Diego, 2008.

4. Côte, A. P.; Benin, A. I.; Ockwig, N. W.; O’Keeffe, M.; Matzger, A. J.; Yaghi, O. M. Porous, Crystalline, Covalent Organic Frameworks. Science 2005, 310 (5751), 1166-1170.

5. Andreas Korinek (2020). Gatan Digital Micrograph file reader (https://www.mathworks.com/matlabcentral/fileexchange/45933-gatan-digitalmicrograph-file-reader), MATLAB Central File Exchange. Retrieved March 18, 2020.

6. Murthy, A. A.; Stanev, T. K.; dos Reis, R.; Hao, S.; Wolverton, C.; Stern, N. P.; Dravid, V. P. Direct Visualization of Electric-Field-Induced Structural Dynamics in Monolayer Transition Metal Dichalcogenides. ACS Nano 2020, 14 (2), 1569-1576. 